\title{
DESARROLLO Y VALIDACIÓN DEL AUTOINFORME DE ADOLESCENTES: ADA
}

\section{DEVELOPMENT AND VALIDATION OF ADOLESCENT SELF-REPORT: ADA}

\author{
Lilian R. Daset * \\ María Eugenia Fernández-Pintos*, ** \\ Daniel Costa-Ball* \\ Concepción López-Soler ${ }^{* * *}$ \\ Wouter Vanderplasschen** \\ *Universidad Católica del Uruguay \\ **Universidad de Gante, Bélgica \\ ***Universidad de Murcia, España
}

\begin{abstract}
Resumen: El Autoinforme de Adolescentes (ADA) evalúa estructuras psicopatológicas y aspectos resilientesprosociales, integrado en un protocolo con una Encuesta Sociodemográfica, de Hábitos y Comportamientos Adictivos a sustancias, internet y videojuegos y variables de salud. Se administró a adolescentes (N=362; 12 a 18 años, ambos sexos) por muestreo intencional no probabilístico en 6 instituciones de nivel secundario de Montevideo. Se analizó por AFE para indicadores categóricos y rotación oblicua, obteniéndose una estructura de 6 factores de banda estrecha, Síndromes de: Depresión-Ansiedad; Disocial con comportamiento adictivo; Desregulación Disruptiva del Estado de Ánimo y Comportamiento Disocial; Ansiedad Social; ResilienciaProsocialidad y de Obsesión-Compulsión. Los Síndromes de Banda Ancha se conformaron por uno que reúne la psicopatología y otro de Resiliencia-Prosocialidad. Los estudios de validez y fiabilidad indican muy buenos índices de bondad de ajuste y adecuados coeficientes estandarizados en las seis dimensiones y una Fiabilidad adecuada. Se concluye de este estudio exploratorio y preliminar que, el Autoinforme ADA ofrece un medio confiable y valido para evaluar los fenómenos descriptos en estas poblaciones. Se iniciará la fase de estudio poblacional con el objetivo de colaborar en la detección de síntomas psicopatológicos emergentes en adolescentes y para determinar los factores de protección.
\end{abstract}

Palabras Clave: estudio instrumental; evaluación psicológica; adolescentes; psicopatología; síndromes empíricos

\begin{abstract}
The Adolescents Self Report (ADA) assess psychopathological structures and resilient-prosocial aspects, integrating a protocol with a sociodemographic survey, Habits and Addictive Behaviors, Internet and video games use and health variables. Was administered to adolescents ( $\mathrm{N}=362$; $12-18$ years old, both sexes) by non-probability sampling in 6 private secondary education institutions of Montevideo. It was analyzed by AFE (MPlus) for categorical indicators and oblique rotation, resulting in a structure of six factors narrowband syndromes: depression-anxiety; Conduct with addictive behavior; Deregulation Disruptive Mood and Dissocial Behavior; Social Anxiety; Resilience-Prosociality and obsession-compulsion. Broadband Syndromes are: one made up of psychopathological syndromes and other Resilience-Prosociality. The validity and reliability studies indicate goodness of fit indices and appropriate standardized coefficients in the six dimensions and adequate reliability. The conclusion of this exploratory and preliminary study shows that the Adolescent Self report (ADA) provides a reliable and valid for assessing the phenomena described in this population. A largeepidemiological scale study is launched in order to assist in the detection of emerging psychopathological symptoms in adolescents and to identify protective factors.
\end{abstract}

Keywords: instrumental study; psycological assessment; adolescents; psychopatology; empirical syndromes

\section{Introducción}

En este manuscrito se desarrollan los pasos de construcción de un instrumento de evaluación psicológica y presentan los resultados preliminares del estudio piloto con el que se lo puso a prueba, con el objetivo de tener un Autoinforme que permita elaborar un perfil de los adolescentes; considerando las características de su cultura y aspectos comunes en Iberoamérica.
Las razones de este estudio pueden resumirse en: la necesidad de estrategias de abordaje psicosocial de las poblaciones juveniles. Las nuevas implicancias de los aportes de la Psicología basada en la Evidencia (Daset \& Cracco, 2013; Holmbeck et al., 2008; Hunsley \& Mash, 2005; Youngstrom, 2013) donde se pone el énfasis en las acciones tendientes a incrementar la validez y una evidencia de calidad que posibilite su réplica en otras poblaciones, lo que comienza a incor- 
porarse en la evaluación. Un tercer argumento lo constituye la importancia que han adquirido las técnicas de evaluación llamadas de cribado o screening, permitiendo identificar sujetos y poblaciones en riesgo.

Una cuarta razón refiere al acervo acumulado de estudios sobre niños y adolescentes y los esfuerzos de múltiples grupos de investigación, que han permitido contar con dos insumos sustanciales a la hora de pensar en un desarrollo instrumental: por un lado una metodología de estudio que posibilita "recrear" en forma bastante eficiente la forma de obtención de perfiles poblacionales (Daset, López Soler \& Hidalgo, 2009; Rescorla et al. 2011; Veerman \& De Meyer, 2012). En segunda instancia, los productos traducidos en estructuras sindrómicas robustas que tienden a replicarse en variadas culturas y posibilitan reconocer algunas comunalidades y particularidades y a partir de allí emprender nuevos desarrollos (Achenbach, Dumenci \& Rescorla, 2002; Meehl, 2001; van der Ende, Verhulst, \& Tiemeier, 2012).

El "gold standard" indicaría que en el proceso de evaluación psicológica se deben incluir la observación, entrevistas, cuestionarios e inventarios (una aproximación con múltiples métodos de estudio); se deben atender diferentes niveles de análisis y dimensiones con información proporcionada por el sujeto y familiares, pares, profesores y profesionales. Este debería ser el proceso para la diagnosis, pero resulta un sistema complicado, costoso, lento y que alcanza a un escaso número de jóvenes y sobre el que hay poca evidencia sobre su eficacia (Johnston \& Murray, 2003); a esto se suman las necesidades concretas del profesional.

La Comisión Internacional que trata sobre los Tests (International Tests Commission, ITC) y las organizaciones que promueven los estándares para el desarrollo, traducción, adaptación y uso (AERA, APA y NCME ${ }^{1}$ ), en sucesivas comunicaciones destacan la importancia de la construcción de tests para la medición psicológica adaptados a la cultura, atendiendo a las características lingüísticas de los sujetos en estudio y a aspectos idiosincráticos de la población objetivo (Evers, 2012; Evers, Muñiz, Hagemeister, Høstmælingen, Lindley, Sjöberg \& Bartram, 2013; Muñiz, Elosua \& Hambleton, 2013). Como señalan Carretero-Dios y Perez (2005) el desarrollo instrumental incrementa el

1 American Educational Research Association (AERA); American Psychological Association (APA); National Council on Measurement in Education (NCME). poder predictivo y la capacidad de generalización, como lo ponen de manifiesto los estudios de Goodman, Renfrew y Mullick (2000); Rescorla et al. (2011), entre otros.

El Autoinforme de Adolescentes -ADA- (Daset, Costa y Fernandez, 2015) procura delimitar las comunalidades inherentes a las estructuras psicopatológicas; que sea accesible a la realidad en que serán evaluados, para posibilitar estudios comparativos en una doble vía (con instrumentos no originarios adaptados y con los desarrollados localmente). Se busca contribuir con un test de evaluación "ágil", que ofrezca un perfil individual o grupal, con recursos de calidad, accesible a los profesionales y con tiempos de administración y análisis relativamente breves. Este manuscrito trata sobre el estudio psicométrico y resultados de la exploración preliminar del ADA, finalizada la fase de desarrollo instrumental.

\section{Prevalencia de problemas mentales en jóvenes}

En la adolescencia se detecta un aumento de la prevalencia en psicopatología; según datos de la OMS (2013) se indica una tasa del $20 \%$ de trastornos mentales (TM) en niños y adolescentes, siendo que en la mitad de estos casos los TM se manifiestan antes de los 14 años de edad. A todas luces un tema de preocupación y sobre el que desde la evaluación psicológica se deben de emprender acciones.

En nuestro país, estudios en la temática han indicado una alta prevalencia de trastornos psicológicos en la adolescencia; tanto en perfiles generales como específicos (Cajigas de Segredo, Kahan, Luzardo, Najson, Ugo y Zamalvide, 2004; Daset, 1998; Daset, 2002; Daset, 2005; Daset et al, 2009; Mels y Trias, 2014; Perez-Algorta, 2001; Viola, Garrido y Varela, 2007). En el estudio realizado por Daset et al (2009) con el instrumento Youth Self Report-YSR- (Achenbach, 1991; Daset, 2005; López Soler, García Montalvo, Pérez López, Brito, Tejerína \& Fernández Ros, 1998) con una muestra de 344 adolescentes escolarizados de nivel secundario de Montevideo, se obtuvieron agrupaciones de síndromes que coinciden con los hallazgos de referencia (Achenbach \& Edelbrock, 1987; Costello, Mustillo, Erkanli, Keeler y Angold, 2003; Goodman et al, 2000). Se destaca que los estudios presentaron mayor peso en los síndromes de depresión-ansiedad para el sexo femenino y en masculino en el 
disocial; con un incremento con la edad de los síndromes de depresión-ansiedad, oposiciónimpulsividad y disocial. Los diez síndromes extraídos fueron: depresión-ansiedad, prosocial, disocial, oposición-impulsividad, alteraciones de pensamiento, quejas somáticas, retraimientoevitación, problemas sociales, temor y un síndrome de inmadurez/inadecuación (Daset, 2005: Daset et al, 2009). El estudio de Viola, Garrido y Varela (2007) con 1374 sujetos en edades entre 6 y 11 años con el Child Behavior Checklist -CBCL- (Achenbach et al, 1991) obtiene una prevalencia de trastornos psiquiátricos del $22 \%$, destacando como síndromes internalizantes el de ansiedad y depresión y como externalizantes el TDA-H y trastornos de conducta. En ambos casos y en otros estudios de grupos específicos con esta metodología y la de Goodman (2010), se reiteran resultados similares para el país y coincidentes en mucho con los de OMS (2009).

A nivel internacional se destaca el clásico estudio epidemiológico de Rutter, Tizard y Whitmore en 1970 en la Isla de Wight (como se citó en Rutter, Giller, \& Hagell, 2000) con 2303 participantes de 10 a 15 años. Padres y educadores completaron cuestionarios sobre el comportamiento de los jóvenes. Se evaluaron dos grupos, uno con indicios de desviación psicológica o psiquiátrica vistos por psiquiatras, el otro por muestreo al azar de población general. El estudio indagó: alienación de los chicos con los padres, dificultades de comunicación, ideas autorreferenciales, ideación suicida, ansiedad, tristeza, sentirse desmoralizado y deprimido y la autodesvalorización. La prevalencia fue del 10\% a los 10 años y del $21 \%$ a los 14 años (Rutter, 1997). El incremento mayor en la adolescencia fue esencialmente de depresión y ansiedad.

Uno de los sistemas más difundidos es el de Thomas M. Achenbach, a partir de sus trabajos pioneros con el CBCL a principio de los años 70'. Entre sus antecesores, se cuentan Ackerson, 1942; Hewit y Jenkins, 1946; Jenkins y Glickman, 1946 (como se citó en Achenbach, 1995; Achenbach y Edelbrock, 1987 y 1991) quienes comenzaron calculando correlaciones entre pares de ítems, buscando distinguir síndromes mediante la lectura clínica y de los criterios estadísticos (Achenbach et al, 2002; 2011). En años recientes se desarrolló un estudio en 25 países (Rescorla et al., 2013) con una muestra de 27,861 adolescentes entre 11 a 18 años y sus padres. Se indagó sobre la con- cordancia y equilibrio entre las respuestas de los adolescentes y sus padres en las escalas $\mathrm{CBCL}$ y YSR; los resultados indicaron que, en promedio, los adolescentes reportaron más problemas que sus padres sobre ellos. A mayor acuerdo entre los puntajes de padres y adolescentes, mayor predicción de efectividad del tratamiento.

Goodman (2010) en Gran Bretaña realizó un estudio longitudinal donde examinó una muestra de 3607 adolescentes -11 a 16 años-, sobre las asociaciones -en ambas direccionesentre salud mental y abuso de sustancias. Se usó el cuestionario Parent Report Strengths and Difficulties (Goodman, 2001) y entrevistas, incluyendo los diagnósticos de los clínicos para trastornos mentales. El abuso de sustancias fue medido por el YSR (Achenbach, 1991), agregando consumo regular de alcohol, tabaco y cannabis. Los resultados indicaron que la presencia de síndromes externalizantes predecía -independientemente- todas las formas de abuso de sustancias, particularmente, tabaco. Los síndromes internalizantes se asociaron solamente al consumo de tabaco, esta asociación desaparecía cuando se incluyó comorbilidad con problemas externalizantes. El trabajo de Goodman ha sido continuado en varios estudios (Janssens \& Deboutte, 2009; Richter, Sagatun, Heyerdahl, Oppedal \& Roysamb, 2011).

Navarro-Pardo, Moral, Galán y Beitia (2012) estudiaron la incidencia de diversos trastornos psicopatológicos en pacientes derivados desde pediatría -588 sujetos de 1 a 18 años- y su relación con edad y género, bajo el criterio de presencia de algún trastorno psicopatológico. Se evaluó mediante entrevista clínica, historia clínica y cuestionarios. Los resultados para edades de 12 a 15 años indicaron la prevalencia de trastornos de conducta (TC) y ansiedad (TA) y de 16 a 18 años principalmente TC. En varones prevalecen TC, mientras que en mujeres los TA. También se encontró mayor incidencia de trastornos externalizantes en varones e internalizantes en mujeres.

Costello, Mustill, Erkanli, Keeler y Angold (2003) realizaron un estudio longitudinal con el objetivo de evaluar la prevalencia y desarrollo de TM desde 9 a 16 años de edad ; analizaron una muestra de 1420 sujetos evaluados anualmente según el DSM IV hasta los 16 años. Los resultados indicaron que un $36.7 \%$ (31\% chicas y $42 \%$ varones) tuvo al menos un TM durante el tiempo del estudio. Algunos TM específicos aumentaron prevalencia con la edad: ansiedad social, pánico, depresión y abuso de sustancias. 
Este acercamiento a la compresión de los problemas de salud mental en poblaciones juveniles, muestra la necesidad de contar con sistemas de evaluación que permitan abarcar múltiples variables y atender a la fase de cambios rápidos de esta etapa. Así mismo, aportar a una vieja discusión sobre la calidad de los criterios estadísticos para comprender estructuras psicopatológicas; algo de la controversia que señalan Belloch, Sandín y Ramos (2009) en conceptos como frecuencia, continuidad y la ineludible referencia al constructo de normalidad-anormalidad.

Se presenta el proceso de desarrollo instrumental de ADA, además de informar sobre los resultados del estudio preliminar con una población juvenil; el nivel de confiabilidad de la escala y la validez interna, a la vez que se plantean alcances y limitaciones que se apreciaron. El Objetivo del Proyecto fue desarrollar un Instrumento de Evaluación para Adolescentes que permita obtener un perfil general en lo relativo a aspectos psicopatológicos, sociodemográficos y sobre características resilientes y prosociales.

\section{Método}

\section{Participantes}

Se administró el cuadernillo ADA (Daset et al., 2015) a 362 adolescentes (218 sexo femenino y 144 sexo masculino), con edades comprendidas entre 12 y 18 años $(M=14.61 ; D E=1.23)$, procedentes de 6 liceos seleccionados por muestreo intencional de la totalidad de instituciones de educación secundaria privadas de la ciudad de Montevideo. El $90.4 \%$ eran estudiantes en educación media básica (primero $=132$, segundo $=100$ y tercero $=79$ ) y el $9.3 \%$ en educación media superior (cuarto, quinto y sexto año). De acuerdo con el índice de Nivel Socioeconómico -INSE- (Llambí \& Piñeiro, 2012) que se utiliza para clasificar hogares (según su capacidad de consumo) los niveles socio-económicos se distribuyen en: $59 \%$ de clase Media, $38 \%$ de clase Baja y $2,4 \%$ de clase Alta. El $62.1 \%$ de los adolescentes viven con ambos progenitores y el $32.9 \%$ con uno de los dos. En relación al nivel educativo de los padres, el $1.5 \%$ no terminó su educación primaria, 9.5\% completó primaria, $26 \%$ no finalizó educación media o educación media técnica-profesional (secundaria), 39\% completó la educación media superior o la educación media técnica-profesional, 24\% finalizó educación terciaria universitaria.

\section{Instrumentos}

Características generales del Autoinforme de Adolescentes (ADA)

El Autoinforme de Adolescente - ADA- (Daset et al., 2015) consta de una sección de 118 ítems (117 clínicos +1 "necesito ayuda psicológica") y una encuesta sociodemográfica. La valoración se realiza a partir de la autopercepción del adolescente y la referencia de comparación es con pares. El cuestionario indaga psicopatología y aspectos relativos a la prosocialidad, resiliencia, teniendo en cuenta a su vez, fortalezas, afrontamiento y deseabilidad social. Las respuestas se recogen con una escala Likert de 5 niveles, donde 1 significa nada y 5 siempre (Lozano, García-Cueto y Muñiz, 2008).

La información referente a datos generales y de contexto se recoge con una Encuesta Sociodemográfica sustentada, por un lado, en la encuesta del Índice de Nivel Socioeconómico (INSE) realizada por el Centro de Investigaciones Económicas -CINVE- (Llambi \& Piñeiro, 2012) basado en la encuesta del Instituto Nacional de Estadística del Uruguay y por el otro, surge de encuestas sociodemográficas perfeccionadas por el equipo de la Línea de Investigación (Daset et al., 2013). El Cuadernillo se nomina como ADA con Sociodemo y Encuesta Consumo A y $\boldsymbol{M}$, donde la primer parte está formada por la Encuesta Sociodemográfica (Sociodemo); en segundo lugar se incluye el apartado que refiere al ADA propiamente dicho y en tercer lugar se encuentran las dos Encuestas sobre Consumo de Sustancias (marihuana y alcohol). Al inicio hay unas breves explicaciones de lo que es el conjunto y luego en cada sección se presentan las respectivas consignas.

Los aspectos diferenciales que aporta este instrumento, además de los reactivos para el estudio psicopatológico, refieren a la inclusión de variables sociodemográficas con referente/país, conteniendo las condiciones del hogar y hábitos. Contiene también ítems sobre comportamientos adictivos, autolesión, uso de internet y juegos, aspectos salugénicos, relacionamiento familiar y resolución de problemas. Considera conductas de riesgo, acoso, resiliencia, fortalezas y prosocialidad; indaga además sobre prosocialidad y resiliencia. En este estudio se presentan los análisis realizados con el Autoinforme ADA y de la Encuesta Sociodemográfica solo se toman la edad, sexo, año de curso y las variables que el INSE plantea para determinar el nivel socioeconómico. 
Desarrollo del Autoinforme de Adolescentes (ADA)

El desarrollo del Autoinforme de Adolescentes -ADA- (Daset et al., 2015) se inscribe en la Línea de en Investigación en Niñez y Adolescencia, que tiene como cometido indagar en estas etapas evolutivas a partir de tres ángulos: psicopatológico, sociodemográfico y aspectos positivos en los que se engloba la prosocialidad y resiliencia. En etapas posteriores se agregaron los estudios sobre comportamiento adictivo (Fernandez, Daset \& Vanderplasschen, 2015; Vanderplasschen, Bloor \& McKeganey, 2010), hábitos en el uso de internet y videojuegos (Echeburúa y De Corral, 2010). Se han realizado estudios siguiendo la metodología propuesta por Achenbach et al (1987, 1991) y Goodman et al (2001); los resultados subrayaron la importancia de trabajar con este tipo de abordajes porque posibilitan: elaborar un perfil poblacional que es de utilidad para instituciones educativas, de salud y de ayuda social (Costa-Ball, González-Tornaría, del Arca, Masjuan, \& Olson, 2013); con un proceso con unos costes menores comparados con los sistemas tradicionales, en poblaciones sobre las que se requieren acciones de corto y mediano plazo por sus altos niveles de riesgo. El proyecto se organizó en fases, en la primera se hicieron las revisiones bibliográficas sobre aspectos relativos a problemas mentales en adolescentes a partir del análisis de investigaciones en la materia, atendiendo a: los instrumentos de evaluación y sus características y los resultados en poblaciones juveniles. Los criterios para la revisión bibliográfica sistemática (RS) fueron, de inclusión: estudios con adolescentes; con técnicas de evaluación psicológica y psicopatológica; con resultados en los que constaran la validez y fiabilidad; entre los años 2000 y 2013. Muestras de población general y clínica. Idiomas: inglés, español, portugués. De exclusión: Adultos o mayores de 21 años y Niños menores de 9 años. Estudios con instrumentos de evaluación cualitativa. Estudios sin validación psicométrica alguna. Las palabras clave y sus combinaciones fueron: 1. Adolescentes o Jóvenes y 2. Psicopatología, Taxonomías, Síndromes empíricos, factores riesgo, vulnerabilidad 3 . Resiliencia, Bienestar psicológico, Calidad de vida, Factores de protección y 4 . Consumo de alcohol y marihuana, uso de sustancias, drogas. Las bases consultadas: APA, SCIELO, ISI, SCOPUS, EBSCO, Medline, Pub Med, Web of Science, Portal Timbó; en la Tabla 1 se resumen los resultados obtenidos.

Tabla 1

Principales instrumentos estudiados en el desarrollo del ADA según $R S$

\begin{tabular}{|c|c|c|}
\hline TÉCNICA & AUTOR & AÑO \\
\hline Youth Self-Report (YSR-UY) & Achenbach, T.M y Rescorla, L.A & 1991 \\
\hline Child Behavior Checklist CBCL & Achenbach, T. M & 1991 \\
\hline Strengths and Difficulties Questionnaire SDQ & Goodman & 1997 \\
\hline Fear Survey Schedule for Children (FSSC-R). & Scherer y Nakamura's & 1968 \\
\hline Barkley Deficits in Executive Functioning Scale - Children and Adolescents & Barkley & 2012 \\
\hline Escala de Síntomas de TEPT para Niños y Adolescentes (CPSS) & Foa, Johnson, Feeny y Treadwell & 2001 \\
\hline Cuestionario sobre intimidación y maltrato entre iguales en las aulas (Bullying) & Ortega, R, Mora-Merchán, J.A. y Mora, J & 1995 \\
\hline Escala de Resiliencia Adolescente & Oshio et al. & 2002 \\
\hline Inventario de Depresion (BDI) & Beck, A, Steer, R, y Brown, G & 1996 \\
\hline Inventario de Ansiedad (BAI) & Beck, A & 1993 \\
\hline Inventario de Personalidad (EPQ) & Barret y Eysenck & 1984 \\
\hline Cuestionario de Personalidad para Niños CPQ & Porter, R. B y Cattell, R. B. & 1981 \\
\hline Alcohol use Disorder Identification (Audit) (OMS) & Saunders, Aasland, De la Fuente, y Grant & 1993 \\
\hline Severity of Dependence Scale (SDS) & Gossop, M & 1995 \\
\hline Encuesta consumo de Alcohol & Junta Nacional de Drogas & 2011 \\
\hline Cannabis Abuse Screening Test (CAST) & Beck y Legleye & 2003 \\
\hline Symptom Checklist 90 Revised (SCL-90-R) & Derogatis, L. R & 1994 \\
\hline Cuestionario de Depresión para Niños (CDS) & Lang, M y Tisher, M & 1990 \\
\hline Cuestionario factorial de la personalidad (16-PF) & Cattell, R.B..Cattell, A.K.S y Cattell, H.E.P. & 1993 \\
\hline Escala de Bienestar Psicológico en Jóvenes (BIEPS-J) & Casullo, M.M. y Castro & 2002 \\
\hline Cuestionario de Auto-Control infantil y Adolescente (CACIA) & Capafóns Bonet, A y Moreno, F. S & 2001 \\
\hline Inventario a Situaciones y Respuestas de Ansiedad (ISRA) & Miguel Tobal; J.J.y Cano-Vindel, A.R. & 1988 \\
\hline Inventario Clínico de Millon para Adolescentes (MACl) & Millon, $\mathrm{T}$ & 1992 \\
\hline Escala de Bienestar Psicológico (EBP) & Sánchez-Cánovas, J & 1998 \\
\hline Escala Obsesivo Compulsivo (Y-BOCS) & Yale-Brown & 1994 \\
\hline Escala de Resiliencia (RESIL W\&Y) & Wagnild, G. Young, H. & 1993 \\
\hline Spence Children's Anxiety Scale (SCAS) & Spence, $\mathrm{H}$ & 2010 \\
\hline Inventario de expresión de ira estado-rasgo en niños y adolescentes (STAXI-NA) & Spielberg, C.D & 2009 \\
\hline
\end{tabular}


Las conclusiones de esa RS permitieron, conjuntamente con los estudios que ya se llevaban a cabo en la Línea de Investigación, discriminar algunos de los temas centrales sobre los que se proyectaba indagar: Ansiedad; Depresión; Autolisis; Autolesión; Quejas somáticas; TDAH; Problemas de Pensamiento; Antisocialidad; Problemas de Ingesta; Problemas del Sueño; Comportamiento Adictivo; Abuso-Bullying; Autorregulación/Autocontrol; Bienestar Psicológico; Prosocialidad y Resiliencia.

Se construyó un Banco de Ítems conformado por 958 reactivos, agrupados por temas; se hizo el primer análisis a la luz de los sistemas de clasificación: Manual Diagnóstico y Estadístico de los Trastornos Mentales de la Asociación Americana de Psiquiatría - APA- en su $4^{\mathrm{a}}$ versión revisada -DSM-IV-TR (APA, 2002), la $5^{\text {a }}$ versión-DSM-5- (APA, 2013) y la Clasificación Internacional de Enfermedades de la Organización Mundial de la Salud-OMS- en la $10^{\mathrm{a}}$ versión -CIE-10- (OMS, 1992). Se eliminaron ítems según los siguientes criterios: juicio clínico, redundancia y no pertinencia del ítems; se obtuvieron 145 reactivos que fueron los iniciales de la primer protocolización.

En la fase siguiente se realizó una revisión del protocolo por parte de 5 jueces expertos (experiencia en evaluación psicológica y desarrollo/ adaptación instrumental e investigación) alcanzando un acuerdo interjueces del 92\%. Nuevamente se analizó protocolo y se procedió a trabajar bajo la dinámica de Grupo Focal con 11 jóvenes de entre 12 y 17 años, con el objetivo de analizar: comprensibilidad; calidad de las consignas; pertinencia de tiempos y formatos y qué entendían que buscaba indagar cada reactivo (se usaron para ello 2 protocolos). Al final y tras un descanso, se propuso una instancia de discusión grupal donde se expusieron las consideraciones que merecía el ADA y se generó un diálogo sobre asuntos que fueron relevantes o sobre los que a su entender no había ítems. La valoración fue positiva; en razón de los planteos se reformularon algunos reactivos, especialmente por el uso de términos más propios de los destinatarios, se quitaron los iterativos y otros que generaban incomodidad.

Una vez finalizada la fase anterior se realizó un análisis de todos los insumos obtenidos y se elaboró un protocolo con 117 ítems y una encuesta sociodemográfica y de aspectos de salud, vínculos, resolución de problemas en el hogar y uso de internet y videojuegos. Se incluyen además dos breves encuestas sobre consumo de alcohol y cannabis, la Escala de Consumo de Alcohol (Junta Nacional de Drogas, 2012) y el Test para el cribado de abuso de cannabis -CAST- (Beck \& Legleye 2003). En el desarrollo instrumental se siguieron las directivas de la EFPA (Muñiz, Elosua \& Hambleton, 2013), atendiendo especialmente a:

- Corrección lingüística atendiendo a los modismos de los jóvenes -con el debido cuidado entre el argot y el uso adecuado del idioma- y términos ambiguos;

- Adecuación Práctica, que implicó indagar sobre: formato del instrumento, tiempo y aspectos de la administración y su adecuación práctica;

- Reconocimiento de las reacciones que provoca el protocolo ADA;

- Estimar la capacidad explicativa de las consignas y adecuar su extensión;

Incluir nuevos ítems;

- Obtener un primer resultado de las respuestas que ofreciera el grupo a los ítems;

Indagar sobre rechazos o reactivos que les resultaran incomprensibles o molestos.

Esta fase permitió además, elaborar un instructivo de trabajo los administradores del protocolo ADA; formar a quienes administran la técnica con 3 sesiones extensas teóricoprácticas, previas al estudio piloto; organizar un setting con protocolos, los materiales requeridos y los sistemas de registro.

\section{Procedimientos}

Para la construcción del cuestionario de autoreporte ADA (Daset et al., 2015) y el estudio de sus propiedades psicométricas se han seguido las normas para el desarrollo y revisión de estudios instrumentales (Carretero-Dios y Pérez, 2007). Se desarrollaron las seis primeras -de las siete fases de la normativa- a saber: justificación del estudio, delimitación conceptual del constructo a evaluar, construcción y evaluación cualitativa de ítems, análisis de los ítems, estudio de la dimensionalidad del instrumento o estructura interna, estimación de la fiabilidad y obtención de evidencias externas de validez (Muñiz, Elosua y Hambleton, 2013; Carretero-Dios y Pérez, 2005). Este Proyecto recibió el aval ético de la Universidad y se trabajó con los Consentimientos Informados de Padres, institución y los propios jóvenes.

Participaron estudiantes de enseñanza media básica y medio superior. Los estudios secundarios en el país están estructurados en un ciclo básico (3 años) y uno superior (3 años) 
-post- primario- para edades de 12 a 18 años. Se administró en grupos originales de curso en la institución educativa, por un graduado en Psicología y estudiantes avanzados entrenados. Se aseguró anonimato de los adolescentes. El tiempo promedio de administración fue de 65' y las respuestas se recogieron -manuscritas- en un protocolo impreso individual.

\section{Análisis de datos}

En función de los objetivos se analizaron los ítems del ADA (Daset et al., 2015) para encontrar la estructura factorial subyacente a través de un modelo del análisis factorial exploratorio (AFE); procurando identificar los factores latentes que subyacen a los ítems estudiados (Lloret-Segura, Ferreres-Traver \& Tomás-Marco, 2014; Matsunaga, 2010).

A continuación se define el tamaño muestral adecuado para ítems de naturaleza ordinal y con una matriz de datos de tipo policórica, que simultáneamente sea consistente con el uso de un software apropiado a la naturaleza de los ítems con formato Likert (Lloret-Segura et al., 2014). Se recurrió al criterio de asimetría extrema para valores $\geq 3$ y superiores a 21 para la curtosis (Kline, 2005; Livacic-Rojas, Vallejo \& Fernández, 2006). Se realizó un análisis de ítems (Tabla 2) donde se observa que el $20.4 \%$ de los ítems presentan una asimetría severa y el $9.2 \%$ una curtosis severa; un $9.2 \%$ de los ítems, además, muestran severidad en ambas medidas. Estos valores de asimetría y curtosis evidencian que los ítems no presentan distribución normal. Autores con un criterio estricto consideran que, los ítems se comportan con distribución normal cuando los valores de los coeficientes de asimetría y curtosis se encuentran dentro del rango entre -1 a 1 (Ferrando \& Anguiano-Carrasco, 2010; Muthén \& Kaplan, 1985, 1992); por su parte, autores menos estrictos consideran aceptable el rango -2 a 2 (Lloret-Segura et al, 2014). Cuando la asimetría supera valores de $+/-3$ y la curtosis se ubica en +/-21 se está ante coeficientes extremos, por este motivo, para determinar la matriz de datos a usar con ítems de respuesta tipo Likert y ordinales, con distribuciones no normales (ver Tablas 2 y 3 ) se optó por usar la matriz de correlaciones policóricas (Lloret-segura et al., 2014). Dado que en el análisis de ítems se destaca la ausencia de normalidad, si bien el programa FACTOR utiliza el método de extracción factorial de mínimos cuadrados no ponderados (ULS), que no requiere que se cumplan los supuestos de normalidad multivariada; hay autores que señalan que valores extremos de asimetría podrían afectar la estimación de los parámetros (Muthén, du Toit, \& Spisic, 1997).

Se analizó la matriz de correlaciones policóricas y se estudiaron las distribuciones bivariadas de cada par de ítems, para identificar patrones de relaciones no lineales entre los ítems (Pérez \& Medrano, 2010). Estos patrones violarían el supuesto de linealidad del AFE y de nuevo añadirían "ruido" a la matriz a analizar y confusión a la estructura factorial identificada (LLoret-Segura et al, 2014). Una vez que se seleccionó la matriz a factorizar, se comprobó su grado de adecuación al AFE; la medida KMO es la más habitual (Lloret-Segura et al, 2014).

En relación al método de estimación de factores, se adaptó a las características de los ítems, ordinales y sin distribución de normalidad, caso en que se recomienda aplicar el método de estimación de mínimos cuadrados ponderados, ajustado por la media y la varianza (WLSMW, por sus siglas en inglés Weighted Least Squares Mean and Variance Adjusted) (Flora \& Curran, 2004; Muthén y Muthén 1998-2007). Cuando los datos son ordinales y con dos a cinco categorías de respuestas, se aconseja usar métodos de estimación de parámetros robustos: LS, WLS o Bayesiano, porque producen estimaciones de los parámetros más precisas que los de estimación para datos continuos ML (Schmitt, 2011).

Tabla 2

Valores de asimetría y curtosis

\begin{tabular}{cccccc}
\hline Asimetría & Frecuencia & Porcentaje & Curtosis & Frecuencia & Porcentaje \\
\hline$<-3$ & 0 & 0 & $<-21$ & 0 & 0 \\
-3 a -2 & 0 & 0 & -21 a -3 & 0 & 0 \\
-2 a -1 & 5 & 5.1 & -3 a -0.75 & 13 & 13.3 \\
-1 a 1 & 33 & 33.7 & -0.75 a 0.75 & 29 & 29.6 \\
1 a 2 & 27 & 27.6 & 0.75 a 3 & 23 & 23.5 \\
2 a 3 & 13 & 13.3 & 3 a 21 & 24 & 24.5 \\
$>3$ & 20 & 20.4 & $>21$ & 9 & 9.2 \\
\hline
\end{tabular}


Tabla 3

Análisis de ítems, datos descriptivos del ADA

\begin{tabular}{|c|c|c|c|c|c|c|c|c|c|}
\hline Ítem & Media & DE & Asimetría & Curtosis & Ítem & Media & DE & Asimetría & Curtosis \\
\hline 1 & 2,04 & 1,03 & 0,80 & 0,00 & 60 & 2,09 & 1,34 & 0,90 & $-0,54$ \\
\hline 2 & 3,80 & 1,01 & $-0,76$ & 0,12 & 61 & 4,04 & 1,13 & $-1,30$ & 1,04 \\
\hline 3 & 3,89 & 1,06 & $-1,00$ & 0,63 & 62 & 1,54 & 1,01 & 2,17 & 4,16 \\
\hline 4 & 2,02 & 1,24 & 1,07 & 0,07 & 63 & 1,16 & 0,52 & 4,01 & 18,52 \\
\hline 5 & 2,15 & 1,46 & 0,85 & $-0,82$ & 64 & 1,14 & 0,47 & 3,86 & 15,58 \\
\hline 6 & 2,35 & 1,28 & 0,59 & $-0,85$ & 65 & 1,59 & 1,04 & 1,95 & 3,04 \\
\hline 7 & 1,75 & 1,15 & 1,42 & 0,94 & 66 & 2,15 & 1,32 & 0,92 & $-0,41$ \\
\hline 8 & 1,08 & 0,34 & 5,10 & 3,02 & 67 & 1,72 & 1,09 & 1,46 & 1,20 \\
\hline 9 & 1,39 & 0,94 & 2,58 & 6,00 & 68 & 1,16 & 0,65 & 4,51 & 20,03 \\
\hline 10 & 3,26 & 1,23 & $-0,27$ & $-1,03$ & 69 & 1,29 & 0,82 & 3,16 & 9,52 \\
\hline 11 & 3,83 & 1,25 & $-0,84$ & $-0,38$ & 70 & 3,47 & 1,40 & $-0,53$ & $-1,00$ \\
\hline 12 & 1,61 & 0,95 & 1,83 & 3,12 & 71 & 1,59 & 0,87 & 1,50 & 1,99 \\
\hline 13 & 1,95 & 1,61 & 1,22 & $-0,38$ & 72 & 1,76 & 1,13 & 1,48 & 1,22 \\
\hline 14 & 1,17 & 0,53 & 4,07 & 18,98 & 73 & 1,15 & 0,56 & 4,78 & 25,23 \\
\hline 15 & 1,30 & 0,70 & 2,87 & 8,87 & 74 & 3,75 & 1,32 & $-0,88$ & $-0,39$ \\
\hline 16 & 3,60 & 1,23 & $-0,55$ & $-0,69$ & 75 & 2,08 & 1,31 & 0,92 & $-0,40$ \\
\hline 17 & 1,94 & 1,22 & 1,19 & 0,38 & 76 & 1,23 & 0,72 & 3,75 & 14,24 \\
\hline 18 & 1,75 & 1,09 & 1,55 & 1,62 & 77 & 1,34 & 0,73 & 2,63 & 7,41 \\
\hline 19 & 1,16 & 0,66 & 4,64 & 21,39 & 78 & 1,10 & 0,47 & 5,77 & 35,80 \\
\hline 20 & 2,99 & 1,55 & 0,01 & $-1,51$ & 79 & 1,77 & 1,14 & 1,41 & 0,99 \\
\hline 21 & 1,07 & 0,31 & 5,99 & 42,65 & 80 & 1,47 & 0,93 & 2,08 & 3,60 \\
\hline 22 & 1,80 & 1,23 & 1,44 & 0,87 & 81 & 1,38 & 0,84 & 2,61 & 6,56 \\
\hline 23 & 1,77 & 0,98 & 1,26 & 1,00 & 82 & 1,56 & 1,08 & 2,03 & 3,21 \\
\hline 24 & 2,95 & 1,30 & 0,07 & $-1,08$ & 83 & 1,66 & 1,04 & 1,70 & 2,23 \\
\hline 25 & 1,99 & 1,15 & 0,99 & $-0,02$ & 84 & 1,63 & 1,11 & 1,86 & 2,49 \\
\hline 26 & 1,27 & 0,64 & 3,16 & 11,77 & 85 & 1,09 & 0,43 & 5,98 & 40,60 \\
\hline 27 & 1,05 & 0,36 & 8,75 & 80,61 & 86 & 2,29 & 1,25 & 0,75 & $-0,44$ \\
\hline 28 & 2,22 & 1,18 & 0,71 & $-0,51$ & 87 & 1,67 & 0,98 & 1,52 & 1,67 \\
\hline 29 & 1,10 & 0,44 & 5,51 & 34,80 & 88 & 2,25 & 1,38 & 0,77 & $-0,73$ \\
\hline 30 & 2,04 & 1,10 & 1,03 & 0,45 & 89 & 3,68 & 1,29 & $-0,77$ & $-0,49$ \\
\hline 31 & 1,02 & 0,17 & 8,88 & 84,86 & 90 & 1,77 & 1,11 & 1,36 & 0,73 \\
\hline 32 & 1,67 & 1,00 & 1,58 & 1,81 & 91 & 3,39 & 1,30 & $-0,47$ & $-0,86$ \\
\hline 33 & 1,47 & 0,88 & 2,18 & 4,46 & 92 & 1,94 & 1,24 & 1,19 & 0,28 \\
\hline 34 & 4,24 & 1,04 & $-1,60$ & 2,05 & 93 & 1,30 & 0,69 & 2,63 & 6,94 \\
\hline 35 & 1,88 & 1,22 & 1,28 & 0,52 & 94 & 3,74 & 1,23 & $-0,81$ & $-0,29$ \\
\hline 36 & 3,84 & 1,10 & $-0,93$ & 0,30 & 95 & 2,67 & 1,29 & 0,44 & $-0,88$ \\
\hline 37 & 1,92 & 1,30 & 1,26 & 0,29 & 96 & 2,44 & 1,35 & 0,53 & $-0,92$ \\
\hline 38 & 1,18 & 0,76 & 4,32 & 17,54 & 97 & 1,80 & 1,19 & 1,33 & 0,57 \\
\hline 39 & 3,87 & 1,37 & $-1,07$ & 0,21 & 98 & 1,45 & 0,95 & 2,30 & 4,60 \\
\hline 40 & 3,43 & 1,37 & $-0,42$ & $-1,06$ & 99 & 2,32 & 1,33 & 0,76 & $-0,58$ \\
\hline 41 & 1,27 & 0,79 & 3,24 & 10,05 & 100 & 2,70 & 1,41 & 0,25 & $-1,23$ \\
\hline 42 & 3,72 & 1,14 & $-0,61$ & $-0,58$ & 101 & 1,56 & 0,97 & 1,88 & 3,01 \\
\hline 43 & 1,40 & 0,91 & 2,62 & 6,44 & 102 & 3,95 & 1,33 & $-1,13$ & 0,00 \\
\hline 44 & 2,05 & 1,35 & 1,09 & $-0,13$ & 103 & 2,90 & 1,43 & 0,08 & $-1,29$ \\
\hline 45 & 1,97 & 1,28 & 1,11 & $-0,03$ & 104 & 2,30 & 1,33 & 0,62 & $-0,86$ \\
\hline 46 & 1,52 & 0,99 & 2,17 & 4,13 & 105 & 2,73 & 1,45 & 0,29 & $-1,31$ \\
\hline 47 & 1,09 & 0,34 & 4,19 & 17,74 & 106 & 2,91 & 1,44 & $-0,02$ & $-1,33$ \\
\hline 48 & 1,28 & 0,71 & 3,41 & 13,14 & 107 & 3,30 & 1,47 & $-0,41$ & $-1,24$ \\
\hline 49 & 1,45 & 0,88 & 2,18 & 4,25 & 108 & 1,68 & 1,08 & 1,65 & 19,10 \\
\hline 50 & 1,75 & 1,06 & 1,36 & 1,08 & 109 & 1,57 & 1,06 & 1,92 & 2,73 \\
\hline 51 & 3,52 & 1,23 & $-0,66$ & $-0,53$ & 110 & 2,32 & 1,23 & 0,65 & $-0,50$ \\
\hline 52 & 1,27 & 0,78 & 3,32 & 10,97 & 111 & 1,61 & 1,01 & 1,77 & 2,52 \\
\hline 53 & 1,31 & 0,81 & 2,80 & 7,29 & 112 & 1,80 & 1,25 & 1,44 & 0,81 \\
\hline 54 & 1,21 & 0,61 & 3,36 & 11,98 & 113 & 2,41 & 1,40 & 0,58 & $-1,00$ \\
\hline 55 & 1,20 & 0,62 & 3,50 & 12,65 & 114 & 1,15 & 0,56 & 4,71 & 24,56 \\
\hline 56 & 1,19 & 0,55 & 3,69 & 16,03 & 115 & 1,72 & 1,19 & 1,58 & 1,30 \\
\hline 57 & 1,70 & 1,06 & 1,57 & 1,71 & 116 & 2,39 & 1,51 & 0,58 & $-1,18$ \\
\hline 58 & 3,71 & 1,10 & $-0,79$ & $-0,06$ & 117 & 2,42 & 1,43 & 0,58 & $-1,03$ \\
\hline 59 & 2,10 & 1,26 & 0,94 & $-0,21$ & & & & & \\
\hline
\end{tabular}

Nota. Valores en asimetría y curtosis mayores a 2 , desvío típico menor a 0.5 evidencian que los ítems no se ajustan a una distribución normal. 
Una vez determinada la matriz de correlaciones y el método de factorización, se selecciona el número de factores adecuado a retener y se realizó el análisis paralelo de Horn (1965); estos procedimientos estadísticos se analizan a la luz de la sustancia teórica que adquieren los factores (Carretero-Dios \& Pérez, 2005). Se cuida que los factores cuenten con un mínimo de 3 ítems; se evaluaron índices de ajuste para la dimensionalidad y se estudió si la solución encontrada es la más plausible y parsimoniosa (Lloret-Segura et al, 2014). Se analizó el ajuste del modelo al patrón de los datos; se utilizó la prueba $X^{2}$, el ratio $X^{2} / g l$, el índice RMSEA, el índice de Tucker-Lewis (TLI), el índice de ajuste comparativo $(\mathrm{CFI})$ y el residuo ponderado cuadrático medio (WRMR). Normalmente se considera una ratio $X^{2} / g /$ con valores inferiores a 2, lo que mostraría un buen ajuste. Los índices CFI y TLI varían entre 0 y 1 , donde 0 indica ausencia de ajuste y 1 un ajuste óptimo. Valores de .95 o superiores son considerados excelentes, en tanto el índice RMSEA es óptimo con valores $\leq$ 0.06 y valores $\leq 0.09$ para WRMR (Schreiber, Nora, Stage, Barlow, \& King, 2006).

En cuanto al método de rotación y asignación de ítems a los factores, se usa la rotación oblicua, geomín oblicuo (Llorente-Segura et al, 2014; Schmitt, 2011). Un aspecto que puede hacer variar la interpretación de la solución obtenida es el criterio de asignación de ítems a los factores, donde la práctica común es retener saturaciones que estén por encima de .30 ó .40 según plantean Bandalos y Finney (como se citó en Lloret-Segura et al, 2014); se ha afirmado que .32 podría ser una buena regla general en la saturación mínima a considerar -equivale aprox. al $10 \%$ de la varianza explicada-; otros autores son más estrictos y recomiendan elevar el punto de corte si la muestra es inferior a 300 casos (Williams, Brown y Onsman, 2010). También se sugiere que la discrepancia entre las saturaciones en los dos primeros factores sea $.50 / .20$ ó .60/.20 (es decir, una diferencia de .30-.40). En este estudio -con una muestra mayor a 300 participantes- se opta por $\geq .30$; los ítems que no superan ese valor son sometidos a un examen desde el punto de vista sustantivo y metodológico, para decidir entre tres opciones: eliminar el ítem, revisar el ítem o añadir nuevos ítems que indaguen la característica relacionada con el mismo. Seguidamente, se realizó un nuevo análisis factorial con la escala reducida, tras eliminar esos ítems (Lloret-Segura et al., 2014).
Por último, se analizaron las evidencias de fiabilidad, para ello se utilizó el alfa ordinal, en lugar del habitual alfa de Cronbach que supone que los datos son continuos (Elosua y Zumbo, 2008) y para estimarlo se usó Microsoft Excel. Para los estadísticos descriptivos se recurrió al paquete estadítico SPSS Statisctics 21 (IBM Corporation, 2012); para el análisis Factorial al Mplus 5 (Muthén \& Muthén, 1998-2007), para el cálculo del número de factores a retener el programa FACTOR (Lorenzo-Seva \& Ferrando, 2006).

\section{Resultados}

Se presentan a continuación los resultados del desarrollo y aplicación del ADA (Daset el al., 2015) en un estudio preliminar (prueba piloto) realizado con 362 jóvenes de entre 12 y 18 años, de ambos sexos, en el marco de 6 instituciones educativas de nivel secundario privadas de la Ciudad de Montevideo. En el Estudio 1 se plantean los análisis de las etapas cuatro y cinco (análisis estadístico de los ítems y estudio de la dimensionalidad del instrumento) en el proceso de desarrollo de instrumentos de evaluación psicológica (Carretero-Dios y Pérez, 2005). Tras el estudio cualitativo de los ítems y luego de seleccionar los mejores del total de reactivos disponibles inicialmente, se realiza el primer AFE; el objetivo es analizar si la matriz de datos se adecua a la factorización y obtener una estimación inicial de la cantidad de factores a retener. Seguidamente se eliminan ítems y se pasa al Estudio 2, con un instrumento más reducido, pero con mejores índices de calidad de los ítems. El Estudio 2 se centra en el análisis de la dimensionalidad del instrumento (estructura interna) en los 98 ítems que conforma la estructura factorial, luego de los que se eliminan en el Estudio 1; se hacen también los respectivos estudios psicométricos (validez de constructo y fiabilidad).

\section{Estudio 1}

\section{Análisis estadístico de los ítems}

El objetivo del estudio fue seleccionar el conjunto final de ítems del ADA a partir del análisis de las propiedades psicométricas de los mismos. Todos los ítems fueron sometidos a un análisis descriptivo, en la Tabla 2 se 
informa la media, desviación típica, asimetría y curtosis. Con los resultados obtenidos se procedió a realizar una primera selección de ítems, estudiando específicamente aquellos que presentan un desvío típico reducido (DE $<0.5)$ y aquellos con valores de asimetría o curtosis mayores a +/- 2 unidades. Con ello se pretende suprimir los que generen respuestas demasiado unánimes y poco discriminativas. La toma de decisiones se realiza a la luz del marco teórico desarrollado en apartados anteriores. En relación a los valores de asimetría, el $20.4 \%$ de los ítems presentan valores de asimetría por encima de 3 , mientras que para los valores de curtosis, el $9.2 \%$ presentan valores superiores a 21 (Tabla 3). El 9.2\% de los ítems presentan valores extremos, tanto en asimetría como en curtosis, evidenciando que no se puede usar la matriz de datos de Pearson para realizar el análisis factorial y es entonces que se decide utilizar la factorización con matriz policórica.

\section{Estructura factorial de la escala ADA con los 117 ítems del Protocolo Original}

En primer lugar, se llevó a cabo un análisis factorial exploratorio sobre la matriz de correlaciones policóricas, con el fin de determinar la estructura factorial del cuestionario. Dado que el coeficiente multivariado de curtosis mostró la ausencia de normalidad, ya que la probabilidad asociada a asimetría multivariada es significativa (Mardia, 1970) se interpreta entonces que, la distribución se aleja de la normalidad de manera significativa y por tanto, es un inconveniente crítico para el análisis (Rodríguez \& Ruíz-Díaz, 2008). Lo anterior se confirma con el análisis de ítems que se observa en las Tablas 2 y 3 , donde se reportó que el $33.4 \%$ presentan valores altos de asimetría y curtosis. Por este motivo se optó por realizar un AFE con la matriz policórica, con el método de extracción factorial de mínimos cuadrados no ponderados (ULS, por sus siglas en inglés Unweighted Least Squares) como elección de preferencia en este tipo de situaciones por su robustez y se utilizó el método Promin para la rotación factorial (Lorenzo-Seva, 1999b), junto con el análisis factorial paralelo (PA) de Horn (1965), basado en el mínimum Rank factor analysis (Timmerman \& LorenzoSeva, 2011).

La prueba de esfericidad de Barlett fue significativa $(X 2=13935.8, p<0.00)$ y el índice de Kaiser-Meyer-Olkin (KMO) de adecuación muestral de .69, mostraron una pobre adecuación de los datos para la aplicación de un análisis factorial. El resultado del análisis paralelo (PA) pone en evidencia la adecuación a una solución de siete factores.

Una vez establecida la solución factorial más adecuada se utilizó el método de rotación Promin (Lorenzo-Seva, 1999a), con el objetivo de obtener una solución factorial simple. La matriz de saturaciones de la solución factorial obtenida permite identificar los contenidos de seis factores, explicando entre todos ellos el $33.34 \%$ de la varianza. Los resultados obtenidos, evidencian un índice de bondad de ajuste malo $(C F I=0.43 ; G F I=0.92)$. Con el fin de evaluar el ajuste de esta solución de siete factores, se calculó la raíz media cuadrática residual (RMSR), obteniendo un valor de 0.052 indicando un buen ajuste, dado que el límite superior para considerar un ajuste aceptable según el criterio de Kelly es de 0.066. Dado el índice KMO descendido (0.69) y los valores de bondad de ajuste bajos, no se analiza el resultado de la factorización sugerida por el PA.

Consecuentemente, con los resultados descriptos anteriormente se vuelve a la etapa de validez de contenido, específicamente a las etapas de: delimitación conceptual del constructo a evaluar, análisis de ítems, dimensionalidad y fiabilidad (Carretero-Dios \& Pérez, 2005). Se detectaron ítems con valores extremos de asimetría y curtosis, que una vez analizados desde el punto de vista clínico, pueden ser eliminados por posibles defectos en sus enunciados, ambigüedad y por escaso aporte al constructo. También se eliminaron ítems que, desde la validez de contenido se encontró que no aportaban en forma importante a los constructos que se busca indagar. De esta manera se purificó el banco de ítems original, se eliminaron 19 ítems del protocolo ADA (Daset et al., 2015) pasando de los 117 ítems de la versión inicial a 98 ítems en la versión refinada (se mantiene el que refiere a la necesidad de ayuda).

\section{Estudio 2}

En esta fase se trabaja con la versión refinada de 98 ítems, se realiza un AFE para matriz policórica con el Programa FACTOR (LorenzoSeva \& Ferrando, 2006) con la finalidad de estudiar el número de factores a extraer, en tanto posibilita el análisis paralelo de Horn (1965). Se aplica un AFE, con la matriz de datos policórica 
de los 98 ítems seleccionados de los 117 originales del ADA, con el método de estimación de factores de mínimos cuadrados no ponderados (ULS), con rotación oblicua, aplicando el método Promin de Lorenzo-Seva (1999a). La prueba de esfericidad de Barlett fue signficativa ( $\mathrm{x} 2=$ $13935.8, p<0.00)$ y el índice KMO es de .81, mostrando una muy buena adecuación de los datos para la aplicación de un análisis factorial.

En cuanto a la cantidad de factores a extraer, los resultados del análisis paralelo (PA) de Horn (1965) ponen de manifiesto la posible extracción de seis dimensiones, por lo que la escala global podría ser considerada multidimensional. Se analiza la matriz de datos con un AFE (con Mplus 5) para indicadores categóricos u ordinales, usando el método de extracción de mínimos cuadrados ponderados (Schmitt, 2011), ajustados por la media y la varianza (WLSMW), con rotación geomin oblicua. En la Tabla 4 se puede visualizar el resultado de los AFE para cada modelo propuesto (desde el modelo unidimensional hasta un modelo de siete dimensiones). Se pude ver, atendiendo a los índices de ajuste de cada modelo que, a modelos con mayor cantidad de factores mejor índice de bondad de ajuste, lo que está en consonancia con los resultados señalados.

En base al AP de Horn (1965) se recomienda la extracción de hasta 7 factores, no obstante, siguiendo los principios de parsimonia y la plausibilidad, se opta por la estructura más sencilla, clara y con valores de bondad de ajuste adecuados; se analizan entonces 6 agrupaciones de ítems. Estas 6 dimensiones se estudian desde el punto de vista clínico y en relación a la validez de contenido, se observa que es la solución más adecuada. Seguidamente se eli- minaron los ítems con pesos menores de $.30 \mathrm{y}$ los que tenían una diferencia menor de .15 en dos factores.

\section{Estructura factorial de la escala ADA con 98 ítems}

En la Tabla 5 se presenta la estructura factorial del cuestionario ADA para variables categóricas, con método de estimación WLSMW y rotación geomin oblicua. Los resultados indican muy buenos índices de bondad de ajuste -ver también Tabla 4- y adecuados coeficientes estandarizados en las seis dimensiones. En la salida planteada en la Tabla 5 se no figuran ítems con cargas residuales, que no aportaban al constructo en este estudio y además, dos se repiten en mas de un factor por su contenido. La decisión de eliminar algunos reactivos que han tenido un comportamiento muy estereotipado o clisé y que aportan poco al constructo, deberá ser tomada una vez se realice el estudio poblacional, en tanto es necesario un mayor número de sujetos en algunas de las categorías para tomar esta decisión.

Los valores de Ajuste son:

$$
\begin{aligned}
& X_{(246)}=297,76 ; \quad X 2 / g l=1,51 ; \quad C F=0,94 ; \\
& \mathrm{TH}=0,95 ; \quad \mathrm{RMSEA}=0,038 ; \quad \mathrm{SRMR}=0,067
\end{aligned}
$$

\section{Fiabilidad}

Una vez obtenida evidencia certera de la dimensionalidad, es el momento se estudia la Fiabilidad de cada una de las dimensiones encontradas. La consistencia interna de la versión final del cuestionario ADA fue estimada mediante el coeficiente alfa ordinal; donde los

Tabla 4

Análisis Factorial Exploratorio: índices de ajuste en función del número de factores en cada extracción

\begin{tabular}{ccccccccc}
\hline Factores & $\boldsymbol{x} \mathbf{2}$ & $\boldsymbol{g l}$ & $\boldsymbol{p}$ & $\boldsymbol{\chi} \mathbf{2} / \mathbf{g l}$ & $\mathbf{C F I}$ & TLI & RMSEA & SRMR \\
\hline $1^{* *}$ & 604.832 & 181 & .0000 & 3.34 & .74 & .797 & .081 & .011 \\
$2^{* *}$ & 460.645 & 181 & .0000 & 2.54 & .83 & .866 & .066 & .099 \\
$3^{* *}$ & 373.131 & 191 & .0000 & 1.95 & .889 & .918 & .052 & .082 \\
$4^{* *}$ & 319.013 & 192 & .0000 & 1.66 & .92 & .94 & .043 & .074 \\
$5^{* *}$ & 309.737 & 197 & .0000 & 1.57 & .93 & .95 & .040 & .069 \\
$\mathbf{6}^{* *}$ & $\mathbf{2 9 7 . 7 6 7}$ & 197 & .0000 & 1.51 & .939 & .956 & .038 & .067 \\
$7^{* *}$ & 288.178 & 197 & .0000 & 1.46 & .94 & .96 & .036 & .064 \\
$8^{* *}$ & 283.486 & 198 & .0000 & 1.43 & .948 & .96 & .035 & .061 \\
$9^{* *}$ & 278.830 & 200 & .0002 & 1.39 & .95 & .967 & .033 & .059 \\
$1^{* *}$ & 275.304 & 202 & .0005 & 1.36 & .955 & .969 & .032 & .057 \\
\hline
\end{tabular}

Nota: ${ }^{* *}$ Numero de factores aconsejados con el percentil 95 (Análisis Paralelo); **Número de factores aconsejados con el percentil 99 (Análisis Paralelo). 
Tabla 5

Pesos factoriales de los ítems extraídos

\begin{tabular}{|c|c|c|c|c|c|c|}
\hline ÍTEMS EXTRAIIDOS & $\mathrm{F} 1$ & $\mathrm{~F} 2$ & F3 & $\mathrm{F} 4$ & F5 & F6 \\
\hline Me siento tan mal que quisiera hacerme daño & 0,939 & & & & & \\
\hline Pienso en como quitarme la vida & 0,872 & & & & & \\
\hline Me lastimo el cuerpo a propósito (cortes, heridas, etc.) & 0,84 & & & & & \\
\hline Me obligo a vomitar después de comer & 0,827 & & & & & \\
\hline He deseado estar muerto & 0,637 & & & & & \\
\hline Me siento muy solola & 0,573 & & & & & \\
\hline Me siento triste e infeliz la mayor parte del tiempo & 0,547 & & & & & \\
\hline Siento que nadie me quiere & 0,535 & & & & & \\
\hline Odio mi aspecto & 0,534 & & & & & \\
\hline Pienso que soy un fracaso & 0,52 & & & & & \\
\hline Pienso que necesito ayuda psicológica & 0,507 & & & & & \\
\hline Lloro mucho mas que otra gente de mi edad & 0,482 & & & & & \\
\hline Siento vergüenza de mí mismola & 0,481 & & & & & \\
\hline Aunque otros piensen que soy delgadola yo me veo gordo/a & 0,477 & & & & & \\
\hline Por momentos siento que dará un ataque o me va a pasar algo... & 0,474 & & & & & \\
\hline Tengo ganas de vomitar (sin que el médico encuentre la causa) & 0,39 & & & & & \\
\hline He tenido ataques de pánico & 0,388 & & & & & \\
\hline Cuando estoy mal, me cuesta entender lo que me pasa & 0,388 & & & & & \\
\hline Hay ideas o imágenes que no puedo sacar de mi cabeza y ... & 0,362 & & & & & \\
\hline Tengo miedo de pensar o hacer algo malo & 0,362 & & & & & \\
\hline Me siento demasiado culpable por cosas que hago & 0,354 & & & & & \\
\hline Tengo dolores de cabeza (sin causa medica aparente) & 0,347 & & & & & \\
\hline Mis compañeros me amenazan o ... por teléfono o internet & 0,33 & & & & & \\
\hline Hago sufrir animales si tengo ganas & & 0,953 & & & & \\
\hline He robado cosas o dinero fuera de mi casa & & 0,522 & & & & \\
\hline Cada vez necesito consumir ... para sentir el mismo efecto & & 0,468 & & & & \\
\hline He prendido fuego cosas que no debía & & 0,461 & & & & \\
\hline Destruyo las cosas de los demás & & 0,395 & & & & \\
\hline Debo ser perfecto/a en todo lo que hago & & 0,391 & & & & \\
\hline Me encanta ser el centro de atención & & 0,369 & & & & \\
\hline Sí tengo ganas de romper algo, me cuesta controlarme & & & 0,723 & & & \\
\hline Si alguien me pega primero, empiezo a pegarle y no puedo parar & & & 0,707 & & & \\
\hline Amenazo a los demás & & & 0,682 & & & \\
\hline Discuto más violentamente que la mayoría de los/as jóvenes ... & & & 0,669 & & & \\
\hline Cuando empiezo a insultar, no puedo parar & & & 0,653 & & & \\
\hline Me peleo a golpes más seguido que los demás & & & 0,646 & & & \\
\hline Golpeo a otros/as a propósito & & & 0,628 & & & \\
\hline Cuando me enojo pierdo el control de lo que digo o hago & & & 0,622 & & & \\
\hline Siento ganas de causar algún tipo de daño a otros & & & 0,593 & & & \\
\hline Me comporto como si fuera del sexo opuesto & & & 0,575 & & & \\
\hline Tengo ataques de rabia que no logro controlar & & & 0,535 & & & \\
\hline Tengo la idea de que algo en mi mente no anda bien & & & 0,527 & & & \\
\hline Me gustaría ser del otro sexo & & & 0,506 & & & \\
\hline Me cuesta estar quietola en mi asiento por mucho tiempo & & & 0,432 & & & \\
\hline Cuanto más me mandan a hacer algo, más voy a tardar en... & & & 0,43 & & & \\
\hline Tengo amigos que tienen problemas por el consumo de drogas & & & 0,411 & & & \\
\hline Destruyo las cosas de los demás & & & 0,378 & & & \\
\hline He prendido fuego cosas que no debía & & & 0,374 & & & \\
\hline Cuando estoy entre mucha gente tengo miedo que se burlen ... & & & & 0,678 & & \\
\hline Por temor a que se burlen de mi, no me acerco mucho a la gente & & & & 0,633 & & \\
\hline Me da miedo ir al liceo & & & & 0,58 & & \\
\hline Mis compañeros/as me dejan de lado en todo & & & & 0,515 & & \\
\hline Recibo más insultos o bromas humillantes de las que ... soportar & & & & 0,506 & & \\
\hline Tengo ... de que otras personas me miran mal o hablan mal ... & & & & 0,5 & & \\
\hline Pienso que soy torpe, incapaz o inferior a los/as demás & & & & 0,498 & & \\
\hline Siento miedo en espacios abiertos o en la calle & & & & 0,482 & & \\
\hline Pienso que soy un fracaso & & & & 0,481 & & \\
\hline La mayoría de mis problemas son por culpa de los demás & & & & 0,479 & & \\
\hline Tengo miedo a tener miedo & & & & 0,455 & & \\
\hline Para mi es difícil demostrar lo que siento & & & & 0,4 & & \\
\hline Soy más miedosola que otros chicos/as de mi edad & & & & 0,399 & & \\
\hline Tengo la idea de que los demás me quieren hacer daño & & & & 0,327 & & \\
\hline Cuando tengo un problema, hago lo que puedo para resolverlo & & & & & 0,478 & \\
\hline Me gusta ayudar a los demás & & & & & 0,474 & \\
\hline Pienso que me van a pasar cosas buenas & & & & & 0,47 & \\
\hline Cuando tengo un problema, pienso que hay una solución & & & & & 0,45 & \\
\hline Aunque pasen cosas difíciles, encuentro motivo para sonreir & & & & & 0,437 & \\
\hline Estar con otras personas me hace sentir bien & & & & & 0,414 & \\
\hline Tengo confianza en mi mismola & & & & & 0,389 & \\
\hline Me siento orgullosola por las cosas que hago & & & & & 0,36 & \\
\hline Cuando estoy mal, me ayuda estar con otros (amigos, familiares) & & & & & 0,356 & \\
\hline Pienso que voy a lograr lo que sueño & & & & & 0,353 & \\
\hline Me siento mejor, cuando hablo con otros de mis problemas & & & & & 0,35 & \\
\hline Cuando cometo un error estoy dispuesto/a a reconocerlo & & & & & 0,348 & \\
\hline Siento que la gente me quiere & & & & & 0,342 & \\
\hline Cuando tengo un problema, me sirve oir otros puntos de vista & & & & & 0,335 & \\
\hline Reviso cosas que hago, para comprobar bien ... (cerrar llave) & & & & & & 0,543 \\
\hline Antes de salir de casa tengo que revisar varias veces mis cosas & & & & & & 0,513 \\
\hline Me siento mejor, cuando hablo con otros de mis problemas & & & & & & 0,359 \\
\hline Cuando estoy mal, me ayuda estar con otros (amigos, familiares) & & & & & & 0,358 \\
\hline Repito ... acciones ( tocar algo, lavar manos) eso me tranquiliza & & & & & & 0,353 \\
\hline Evito ciertas cosas, lugares o actividades que me dan miedo & & & & & & 0,345 \\
\hline Tengo miedo que en mi familia se contagien o enfermen & & & & & & 0,345 \\
\hline Se hace difícil prestar atención por ... tiempo y me trae problemas & & & & & & 0,306 \\
\hline
\end{tabular}


valores encontrados oscilaron en el rango de entre .60 y.90 (una sola en .60) como se puede apreciar en la Tabla 6, las restantes dimensiones coinciden con los valores recomendados de .70 a .80 (Carretero-Dios \& Pérez, 2005); incluso en un contexto de investigación la fiabilidad puede ser considerada adecuada en entorno a .70 (Nunnally \& Bernstein, 1994).

Tabla 6

Matriz de intercorrelaciones entre factores y fiabilidad (ordinal)

\begin{tabular}{lcccccc}
\hline & 1 & 2 & 3 & 4 & 5 & 6 \\
\hline 1. Factor & .90 & & & & & \\
2. Factor & 0.143 & .70 & & & & \\
3. Factor & 0.332 & 0.263 & .90 & & & \\
4. Factor & 0.295 & 0.067 & 0.245 & .76 & & \\
5. Factor & -0.113 & -0.110 & -0.011 & -0.066 & .67 & \\
6. Factor & 0.153 & -0.016 & 0.148 & 0.115 & 0.137 & .60 \\
\hline
\end{tabular}

Nota. Los coeficientes alfa ordinal se presentan en negritas a lo largo de la diagonal.

Los resultados mostraron una fiabilidad adecuada para trabajar en investigación en las seis dimensiones del cuestionario ADA de 98 ítems.

\section{Conformación de los Síndromes Empíricos del estudio preliminar con ADA}

Una vez analizadas las diferentes estructuras factoriales a la luz de los criterios estadísticos y clínicos, se optó por una solución de 6 factores - Síndromes Empíricos (SE) para la población general en estudio. Estos SE tienen algunas características particulares, de ellas una que destaca es el número de ítems que los componen -luego de aplicar los criterios planteados en apartados anteriores-; a su vez, el nivel explicativo de la varianza desciende en general entre el primero y el último. En la Tabla 7 se resumen, según se nominan, posteriormente se hace una breve descripción.

Tabla 7

Síndromes Empíricos extraídos del estudio con $A D A$ en adolescentes

\begin{tabular}{cc}
\hline FACTOR & SínDROME \\
\hline 1 & Depresión-Ansiedad \\
2 & Disocial con Comportamiento Adictivo \\
3 & Desregulación disruptiva del estado \\
de ánimo y Comportamiento disocial \\
4 & Ansiedad Social \\
5 & Resiliencia-Prosocialidad \\
6 & Obsesión-Compulsión \\
\hline
\end{tabular}

\section{Síndrome de Depresión-Ansiedad}

Con la mayor carga factorial de toda la estructura, destacan los reactivos que refieren a depresión, los que son además numerosos y severos. En este caso tanto desde el punto de vista clínico como estadístico, el elevado número de ítems abre a la discusión de si los reactivos están informando sobre un mismo asunto y si la estructura no podría mejor definirse en dos factores una vez se cuente con un muestreo mayor y con más sujetos de edades entre 16 y 18 años (donde los estudios informan se incrementa la prevalencia del trastorno). Pueden considerarse además algunos indicadores de Anorexia Nerviosa para DSM-IV-TR (APA, 2002). Con los estudios que se realizan según la CIE-10 (1992) y los avances en atención primaria, coincide en muchos de los indicadores del Trastorno depresivo en la Infancia, donde los componentes de Ansiedad son habituales. En cuanto a los estudios de referencia, contiene varios de los tópicos derivados de sus estructuras, especialmente en lo que respecta a Depresión (Rescorla et al, 2013; Costello et al, 2003; Goodman et al, 2010).

\section{Síndrome Disocial con comportamiento adictivo}

Este factor está conformado por 7 reactivos, donde prima el comportamiento disocial (DSM-IV-TR, 2002; CIE-10, 1992), con significativos indicadores de problemas psíquicos. En este factor se excluyeron ítems con cargas muy cercanas a .30 para seguir el criterio estadístico (carga .29), pero los mismos expresaban o enfatizaban el constructo subyacente: Agresión a personas y animales y Destrucción de la propiedad, las más claras. El Factor también contiene referencias al consumo de sustancias adictivas, lo que en la literatura es una referencia permanente para grupos de jóvenes donde se presentan asociados actos de violencia con consumo (Birhanu, Bisetengn, \& Woldeyohannes 2014; Wongtongkam, Ward, Day, \& Winefield, 2014).

\section{Síndrome de Desregulación Disruptiva del Esta-} do de Ánimo y Comportamiento Disocial

Conformado por 18 reactivos, que se podrían agrupar en: ítems que refieren a la incapacidad de control de impulsos con ira; ítems relacionados con la violencia hacia los demás e ítems que remiten a lo más directamente disocial. Puede tener cierta equivalencia con 
algunos ítems del Trastorno Disocial del DSMIV-TR y DSM-V (2002; 2013) y del CIE 10 (1992) también para Trastorno Disocial. Con una nueva categoría en el DSM V (2013), el Trastorno de desregulación disruptiva del estado de ánimo (DMDD, por sus siglas en inglés), comparte varios indicadores y tiene el sentido de una amplitud de comportamientos desajustados (Roy, Lopes \& Klein, 2014).

Según DSM V (2013) este tipo de trastorno (DMDD) supone un enojo permanente, por un período prolongado en más de un ámbito -hogar, colegio, etc.-. En este caso tenemos la dificultad del control, la desregulación, el enojo y la irritabilidad, en lo que parece ser más de un ámbito; se carece de la medida de temporalidad, lo que supone un elemento a considerar en el protocolo definitivo en tanto la condición en general en estas etapas y para estos desórdenes es de un año. Hay elementos que hacen pensar en un Trastorno Negativista Desafiante - que según la literatura se presenta como un posible predictor de un posible Trastorno Disocial futuro (DSM-IV-TR, 2002) y en el Trastorno Negativista Desafiante y de Oposicionismo (CIE-10, 1992), pero prevalece la expresión de la ira y la inadecuación mas que el desafío; también ítems que remiten al Trastorno por Déficit de Atención con Hiperactividad (DSM-IV-TR, 2002) .

\section{Síndrome de Ansiedad Social}

Este factor está conformado por 14 reactivos que remiten a los síntomas que se generan ante las interacciones sociales, con ítems sobre autodesvalorización y el consecuente temor a la evaluación social (Costello et al, 2003; Chavira, Stein, Bailey y Stein, 2004). Presenta algunos indicadores que también están planteados en el Trastorno de Ansiedad Generalizada y otros en la Fobia Social del DSM IV-TR (2002), pero será mayoritariamente la Ansiedad Social la que lo defina, a la vez que delinea ciertas consecuencias de la misma en la interacción en grupos

\section{Síndrome de Resiliencia-Prosocialidad}

Se agrupan en este Factor dos tipos de ítems: los que refieren a la autoconfianza y la capacidad de resolución de problemas y los que hablan de una buena valoración de los vínculos y buen ejercicio de la sociabilidad. La Prosocialidad es entendida no sólo como la Deseabilidad
Social sino también como el gusto por estar con otros y hacer por otros, con mucho de los actuales componentes que estudia la Psicología Positiva, transcurriendo entonces entre varias conceptualizaciones (Achenbach, 1991; Crick, 1996). Sin duda hay elementos de Deseabilidad Social como lo indican muchos estudios. Otra característica refiere a que varios ítems de este factor, tuvieron una carga negativa en factores que remiten a comportamientos o pensamientos más disfuncionales.

Este SE se conformó en forma casi idéntica en estructuras de 5, 6, 7, 8 y 9 taxones y analizados con diferentes modelos de AFE, lo que ha resultado bastante significativo desde el punto de vista clínico. La división que se produce entre prosocialidad y resiliencia parece no tener unos límites muy precisos aquí y por tanto no se podría considerar esta agrupación de ítems -en su totalidad- para el estudio de la Deseabilidad Social. La inclusión de reactivos que indagan sobre aspectos positivos como resiliencia, prosocialidad, entre otros, supone una línea más innovadora en este tipo de estudios, que claramente requiere una profundización y más instancias para su validación (Cutuli, Gillham, Chaplin, Reivich, Seligman, ... \& Freres, 2013; Lyubomirsky, King \& Dienner , 2005). Coexisten también reactivos sobre bienestar subjetivo (Dienner, Oishi \& Lucas, 2003) especialmente aquellos que refieren a la evaluación que los jóvenes hacen de sus vidas y además, en este factor se conglomeran ítems que remiten a las definiciones más clásicas de resiliencia, como esa capacidad de afrontar las adversidades y salir robustecido de ellas (Peirano, 2013; Wagnild \& Young, 1993).

\section{Síndrome de Obsesión-Compulsión}

Este sexto factor se estructura con ocho ítems, de los cuales cuatro refieren a comportamientos de tipo ritual obsesivo-compulsivo: evitación y comprobación y uno a la ideación propiamente dicha. Se acompañan de tres indicadores bastante conocidos para el profesional clínico, la necesidad de que otros le tranquilicen y los problemas a nivel atencional-dificultades de concentración por irrupción de pensamientos disfuncionales- que esto genera en las demás actividades diarias. Comparte con claridad indicadores con el Trastorno Obsesivo-Compulsivo con comportamiento ritual de la CIE-10 (1992). Las investigaciones de este Síndrome en edades 
tempranas ha aumentando en el último decenio, coincidiendo muchos de los resultados en la importancia de la detección precoz y en que su estructura es muchas veces más confusa que en adultos, no obstante se identifican con claridad los síntomas ansiosos presentes en la evitación y la comprobación (Nelson, Hanna, Hudziak, Botteron, Heath \& Todd, 2015).

La estructura sindrómica de banda estrecha obtenida en el estudio comparte algunas de las conformaciones de factores que resultaron en los estudios de referencia. Se mantiene la estructuración de un síndrome donde confluyen los problemas disociales, otro donde el elemento común lo conforman las conductas violentas hacia los demás y otro donde la depresión es la que mayor peso tiene en toda la estructura. En forma más difusa, pero también coincidente con varios hallazgos referidos, se organizan un conjunto de reactivos referidos a la Ansiedad, expresada como Ansiedad Social o como Síndrome de Obsesión-Compulsión. Es el Síndrome Prosocial y de Resiliencia el que pone una nota diferencial entre las investigaciones tradicionales en la materia, constituyendo un vector a indagar más en profundidad y a partir del que se espera obtener indicios para la conceptualización de factores de protección.

Una vez extraídos los Síndromes de Banda Estrecha, se indagó sobre la conformación de los Síndromes de Banda Ancha, a tales efectos se usó el método de mínimos cuadrados (ULS) no ponderados, forzando la dimensionalidad a dos, rotación oblimin. El resultado es una medida de KMO de 0.70 y la prueba de esfericidad de Bartlett es significativa a nivel de 0.000 y el porcentaje de varianza explicada es del $61 \%$. Las cargas factoriales en la primer dimensión son: Factor 1 ( S. de Depresión-Ansiedad, 0.81), el Factor 4 (S. de Ansiedad Social, 0.68), el Factor 3 ( S. de Desregulación disruptiva del estado de ánimo y comportamiento disocial, 0.60), el Factor 2 (S. Disocial con Comportamiento Adictivo, 0.30) y el Factor 6 - que carga en ambas dimensiones- (S. Obsesivo-Compulsivo, 0.66). En la segunda dimensión se ubica el Factor 5 (S. de Resiliencia-Prosocialidad). Eliminando el Factor 5 o Síndrome de Resiliencia-Prosocialidad de la estructura general, se analiza nuevamente por el mismo método, usando la regla de Kaiser $(K>1)$. La medida de adecuación muestral KMO es de 0.70 y la prueba de esfericidad de Bartlett es significativa para una única dimensión que explica el $49 \%$ de la varianza total. Sería inte- resante poder indagar lo planteado sobre un posible Factor $p$, que refiere a una gran dimensión latente psicopatológica (Caspi, Houts, Belsky, Goldman-Mellor, Harrington, Israel,... Moffitt, 2013; Laceulle, Vollebergh \& Ormel, 2015). Todos estos asuntos son interrogantes a considerar en la próxima fase, el estudio poblacional y de baremación del instrumento ADA.

\section{Discusión y Conclusiones}

En salud mental se transita sobre una constante de multicausalidad y un interjuego entre variables personales, contextuales y la expresión de todo ello en un espacio y tiempo. La evaluación psicológica debe asumir tales desafíos, con un marco de fondo pincelado por los avances de la ciencia y la transversal "era de la evidencia" en que se está ingresando. El desarrollo instrumental del que trata este artículo toma en consideración tal escenario y propone un acercamiento a partir de la construcción de un Autoinforme de Adolescentes, ADA, que tiene como objetivo evaluar aspectos psicopatológicos, sociodemográficos, de prosocialidad y resiliencia en adolescentes; en forma económica y ágil, a nivel de cribado, para obtener un perfil y detectar factores de riesgo y de protección.

El Autoinforme de Adolescentes ADA (Daset et al., 2015) según los estudios consultados, es la primera técnica de cribado de aspectos psicopatológicos y positivos de adolescentes desarrollada en y para nuestro país. No contamos en nuestro medio con un instrumento que indague síndromes psicopatológicos y variables positivas en adolescentes, validado según la normativa internacional; se apoya en los esfuerzos de distinguidos colegas que con su tarea de adaptación y construcción para áreas específicas han colaborado en cimentar este emprendimiento.

Del estudio psicométrico se desprenden algunas limitaciones que se toman como insumo para la próxima fase; en primer lugar, dado el diseño metodológico a partir de una muestra no probabilística, no es posible extraer conclusiones causales ni generalizables al total de la población de jóvenes del país. En segundo lugar la muestra no tuvo la amplitud necesaria en cuanto a edades y nivel de curso secundario al que asisten. Sería así mismo útil generar un protocolo para la inclusión de otro informante-padres, profesores-. En lo que respecta a los ítems, los resultados han generado un nuevo protocolo -final de 98 ítems- el que se esperaría no supere en la fase 
final los 90 reactivos y abreviar la Encuesta Sociodemográfica que lo acompaña. Estos insumos deberán ser considerados en primer término y además, decidir sobre la inclusión de otros instrumentos que indaguen los grupos síndrómicos delineados para la fase de validación externa e indagar sobre otros trastornos que no se han visto representados en estos resultados.

El método de estimación que se utilizó es el de mínimos cuadrados ponderados ajustado por la media y la varianza (WLSMW), para un AFE con variables categóricas ordinales; se necesita entonces de un método de estimación robusto que no requiera normalidad multivariada. En este caso el $9.2 \%$ de los ítems presentan valores de asimetría y curtosis extremas (As $>3$; Cur $>21$ ). Se plantea una línea de investigación a seguir profundizando para analizar la estabilidad de las estimaciones de los parámetros con métodos robustos, frente a ítems con índices de asimetría y curtosis muy altos. En tercer lugar, es recomendable el uso secuencial del AFE y AFC. Estas limitaciones son un tema intrínseco a los estudios en psicopatología y en clínica en general, lo que hace tan imprescindible propuestas como las de la Psicología y la Evaluación basadas en la Evidencia y las de metodólogos, psicómetras y expertos en estadística que, conjuntamente con los clínicos analicen las particularidades de esta área. En el trascurso de esta investigación fueron surgiendo preguntas que necesitaban para su respuesta tanto de los criterios estadísticos como clínicos; en ocasiones la mejor forma estadística no reflejaba la estructura que adquiere el síndrome y menos esa esencia que lo convierte en una entidad en sí mismo.

La estructura factorial que se obtuvo es consistente con muchos de los hallazgos de los estudios de referencia, especialmente en los Síndromes de Depresión-Ansiedad; Síndrome Disocial con comportamiento adictivo; Síndrome de Desregulación disruptiva del estado de animo y Comportamiento Disocial; Síndrome de Ansiedad Social; Síndrome de Resiliencia-Prosocialidad y un sexto factor que se nominó como Síndrome de Obsesión-Compulsión. En el análisis de los Síndromes de Banda Ancha se obtuvo uno que concentra todos los factores de sustrato psicopatológico y un segundo factor con el Síndrome de Resiliencia-Prosocialidad, dejando abierta la discusión y estudio la cuestión de una única dimensión o factor $p$ (psicopatológico) o insistir en el análisis de posibles estructuras que planteen los síndromes internalizantes y externalizantes y aún uno de carácter mixto, como ocurrió en una de las soluciones obtenidas. El elemento común en este estudio es -tanto para una sola dimensión, como para dos y tres dimensionesque por un lado se agrupan los síndromes de composición psicopatológica y por el otro se estructura la resiliencia y prosocialidad; esta es a nuestro entender, una derivación importante a profundizar y para la que se considera incluir preguntas en la encuesta y una escala paralela. Este resultado ¿está planteando la figura de los factores de protección?

Los resultados de los estudios de validez y fiabilidad de este instrumento aplicado a la población mencionada indican muy buenos índices de bondad de ajuste y adecuados coeficientes estandarizados en las seis dimensiones. En cuanto a la fiabilidad se ubica dentro de lo que se criterio como adecuado para trabajar en investigación en las seis dimensiones del cuestionario de 98 ítems. De los resultados del estudio psicométrico se concluye que, el Autoinforme de Adolescentes -ADA- ofrece un medio confiable y valido para estudiar los fenómenos descriptos en esta población en particular y su contexto, permitiendo a partir de los resultados obtenidos desarrollar programas efectivos y específicos enfocados a la prevención psicológica en adolescentes y para potenciar factores de protección.

Este instrumento resulta de utilidad para el ámbito educativo, clínico, social y para la investigación académica; por lo que requiere para su validación definitiva que sea administrado a una población amplia de adolescentes (muestra sugerida de aprox. 2500 sujetos) con los parámetros de edad y sexo del estudio piloto, tanto con población general como clínica. A esto debería sumarse, para el estudio de su validez externa, la evaluación con instrumentos específicos (Depresión, Ansiedad, etc.) y generales de cribado psicopatológico, a efectos de finalizar la fase de desarrollo instrumental y poder contar con un Protocolo de Evaluación que permita cubrir las necesidades básicas de los profesionales en salud mental con estos grupos etarios en Uruguay.

La forma en que se realiza esa búsqueda, la verificabilidad y la reformulación están íntimamente ligadas con el procedimiento usado para obtener las características que definen a los síndromes; el matiz diferencial de este sistema de clasificación obedece al proceso empírico de recogida y análisis de la información sobre similitudes y diferencias, lo que Meehl (2001; 2004) define como el enfrentamiento con un 
número infinito de maneras en que los individuos difieren y la aceptación del desafío de limitarlo a unas estructuras latentes finitas. Los Síndromes derivados empíricamente son fenómenos que tienden a co-ocurrir; la asignación de un individuo a un Síndrome es una cuestión de grado, pudiendo además tener puntuaciones en otros síndromes, esto es lo que la hace tan flexible para la decisión clínica y como plantea Achenbach (1991) cualquier taxonomía debe verse como un medio transitorio para organizar los datos, más allá de que pueda ser prometedora y hasta muy atractiva su conformación a los ojos de los investigadores; es un método, una estrategia y por lo tanto uno de los caminos posibles para la comprensión de fenómenos psicológicos complejos y que debe ser revisada con cuidado y en forma permanente. Una tarea que requiere de análisis estadísticos a la luz de los criterios clínicos, bajo los que cobra sentido.

\section{REFERENCIAS}

Achenbach T. M. (1991). Integrative Guide to the 1991 CBCL/4-18, YSR, and TRF Profiles. Burlington, VT: University of Vermont, Department of Psychiatry.

Achenbach, T. M. \& Dumenci, L.. (2001). Advances in Empirically Based Assessment: Revised Cross-Informant Syndromes and New DSM -Oriented Scales for the CBCL, YSR, and TRF: Comment on Lengua, Sadowksi, Friedrich, and Fisher. Journal of Consulting and Clinical Psychology, 69(4), 699-702.

Achenbach, T. M. (1995). Empirically Based Assessment and Taxonomy: Applications to Clinical Research. Psychological Assessment, 7(3), 261-274.

Achenbach, T. M. (2011). Commentary: Definitely more than measurement error: But how should we understand and deal with informant discrepancies?. Journal of Clinical Child and Adolescent Psychology, 40, 80-86

Achenbach, T. M., Conners, C. K., Quay, H. C., Verhulst, F. C. \& Howell, C. T. (1989). Replication of empirically derived syndromes as a basis for taxonomy of child/ adolescent psychopathology. Journal of Abnormal Child Psychology, 17, 299-323.

Achenbach, T., Dumenci, L. \& Rescorla, L.A. (2002). Tenyear comparisons of problems and competencies for national samples of youth: self, parent, and teacher reports. J. of Emotional and Behavioral Disorders, 4, 215-228.

Achenbach, T.M. \& Edelbrock, C.S. (1987). Manual for the Youth Self Report and profile. Burlington: University of Vermont.

Achenbach, T.M. (1991). Manual for the Youth Self Report and 1991 profile. Burlington VT: University of Vermont.

American Psychiatric Association (2002). Manual Diagnóstico y Estadístico de los trastornos mentales, DSM-IV-TR. Barcelona: Masson

American Psychiatric Association. (2013). Diagnostic and Statistical Manual of Mental Disorders (Fifth ed.). Arlington, VA: American Psychiatric Publishing.
American Psychological Association Presidential Task Force on Evidence-Based Practice: APA. (2006). Evidencebased practice in psychology. American Psychologist, 61, 271- 285.

Beck, F.\& Legleye, S. (2003). Drogues et adolescence. Usages de drogues et contextes d'usage entre 17 et 19 ans, évolutions récentes, ESCAPAD (2002), Rapport OFDT.

Belloch A., Sandín B. y Ramos F. (2009). Manual de Psicopatología. Vol. 1 y 2. .Madrid: McGraw-Hill.

Birhanu, A, Bisetengn \& Woldeyohannes (2014) High prevalence of substance use and associated factors among high school adolescents in Woreta Town, Northwest Ethiopia: multi-domain factor analysis. BMC Public Health, 14: 1186.

Cajigas de Segredo, N.; Kahan, E.; Luzardo, M.; Najson, S.; Ugo, M C \& Zamalvide (2004). Validación de la escala de agresión entre pares (Bullying) en una muestra montevideana de adolescentes de ciclo básico. VII Jornadas de Psicología Universitaria Montevideo, UR, Facultad de Psicología. pp. 452-457.

Carretero-Dios H. \& Pérez C. (2005). Normas para el desarrollo y revisión de estudios instrumentales. International Journal of Clinical and Health Psychology, 5(3),521-551.

Carretero-Dios, H. \& Pérez, C (2007). Standards for the development and review of instrumental studies: considerations about test selection in psychological research. International Journal of Clinical and Health Psychology, 7(3), 863-882.

Caspi, A., Houts, R. M., Belsky, D. W., Goldman-Mellor, S. J.,Harrington, H., Israel, S., . . . Moffitt, T. E. (2013). The $p$ factor: One general psychopathology factor in the structure of psychiatric disorders? Clinical Psychological Science, 2, 119-137. doi:10.1177/2167702613497473

Chavira, D. A., Stein, M.B., Bailey, K. \& Stein, M.T. (2004). Child anxiety in primary care: Prevalent but untreated. Depression and Anxiety, 20, 155-164.

Costa-Ball, D., González-Tornaría, M., del Arca, D., Masjuan, N. \& Olson, D.H. (2013). Propiedades Psicométricas del FACES IV: Estudio de validez en población uruguaya. Ciencias Psicológicas VII (2): 119 - 132.

Costello, E. J., Mustillo, S, Erkanli, A, Keeler, G. \& Angold, A. (2003). Prevalence and development of psychiatric disorders in childhood and adolescence. Archives of General Psychiatry, 60(8), 837-44.

Crick, N.R. (1996). The Role of Overt Aggression, Relational Aggression, and Prosocial Behavior in the Prediction of Children's Future Social Adjustment. Child Development, 67 (5), 2317-2327.

Cutuli, J. J., Gillham, J. E., Chaplin, T. M., Reivich, K. J., Seligman, M.E.P., Gallop, R. J., Abenavoli, R. M. \& Freres, D. R. (2013). Preventing adolescents' externalizing and internalizing symptoms: Effects of the Penn Resiliency Program. International Journal of Emotional Education, 5(2), 67-79.

Daset L.R., Costa-Ball D. \& Fernandez-Pintos, M.E. (2015). Manual del Autoinforme de Adolescentes (ADA). Manuscrito en preparación.

Daset, Fernández-Pintos, Costa, López-Martirena, Benedetti, Peirano, Castellucio, Lariau y Díaz-Saetone, (octubre, 2013). Adolescencia: Acercándonos a un Perfil Uruguayo a partir del Autoinforme de Adolescentes $(A D A)$. IV Simposio de Investigación en Psicología, Universidad Católica del Uruguay. 
Daset, L. R. (1998). Aproximación a una taxonomía empírica: estudio en jóvenes de Uruguay. Comunicación presentada al II Congreso de la Asociación española de Psicología Clínica y Psicopatología. Libro de Resúmenes: 83-84. España..

Daset, L. R. y Cracco, C. (2013). Psicología basada en la Evidencia: algunas cuestiones básicas y unaaproximación a través de una revisión bibliográfica sistemática. Ciencias Psicológicas, 7(2), 209-220.

Daset, L.R. (2002). Depresión en la Adolescencia: una aproximación desde la evaluación empírica. Prisma, 18, 25-32.

Daset, L.R. (2005). Psicopatología en la Adolescencia: relación entre aspectos psicopatológicos, variables sociodemográfica y competencias en una población de jóvenes uruguayos. Tesis doctoral. Murcia. España.

Daset, L.R.; Lopez Soler, C. \& Hidalgo, M.D. (2009). Síndromes empíricos en una población adolescente evaluados mediante el YSR. Ciencias Psicológicas, 3(1), 67-82.

Diener, E., Oishi S. \& Lucas, R. E. (2003). Personality, Culture, and Subjective Well-Being: Emotional and Cognitive Evaluations of Life. Annual Review.of Psychology, 54:403-25.

Echeburúa, E. \& De Corral, P. (2010). Adicción a las nuevas tecnologías y a las redes sociales en jóvenes: un nuevo reto. Adicciones, 22(2), 91-96

Evers A, Muñiz J. Hagemeister, C, Høstmælingen A., Lindley P., Sjöberg A. \& Bartram D.(2013). Assessing the quality of tests: Revision of the EFPA review model. Psicothema Vol. 25, No. 3, 283-291.

Evers, A. (2012). The Internationalization of Test Reviewing: Trends, differences and results. International Journal of Testing, 12, 136-156.

Elosua, P. \& Zumbo, B. (2008). Coeficientes de fiabilidad para escalas de respuesta categórica ordenada. 20 (4), 896-901.

Fernandez, M.E, Daset, L. R. \& Vanderplasschen, W. Risk and protective factors in alcohol and marihuana using adolescents in Montevideo (Uruguay). Ninth annual conference of the International Society for the Study of Drug Policy. Ghent, Belgium, May 2015.

Ferrando, J., \& Anguiano-Carrasco, C. (2010). Sección Monográfica. Papeles de Psicólogo, 31(1), 18-33.

Flora, D. B., \& Curran, P. J. (2004). An empirical evaluation of alternative methods of estimation for confirmatory factor analysis with ordinal data. Psychological Methods, 9(4), 466-491.doi: /10.1037/1082-989X.9.4.466

Goodman, R. (2001). Psychometric properties of the Strengths and Difficulties Questionnaire. Journal of the American Academy of Child and Adolescent Psychiatry, 40, (11) 1337-1345.

Goodman, R. (2010). Substance use and common child mental health problems: examining longitudinal associations in a British simple. Addiction, 105, 1484-1496.

Goodman, R., Renfrew, D. \& Mullick, M (2000). Predicting type of psychiatric disorder from Strengths and Difficulties Questionnaire (SDQ) scores in child mental health clinics in London and Dhaka. European Child \& Adolescent Psychiatry, 9, 129-124.

Greenbaum, P. E. (1998). Hierarchical Confirmatory Factor Analysis of the Child Behavior Checklist/4-18. Psychological Assessment, 10(2),149-155.

Hernández, López,T., Roldan Fernández, J., Jiménez Frutos, A., Mora Rodríguez, C, Escarpa Sánchez-Garnica \& Pérez Álvarez, M. (2009). La Edad de inicio en el consumo de Drogas, un indicador de consumo problemático. Intervención Psicosocial, 18(3), 199-212.
Holmbeck, G.N., Thill, A.W., Bachanas, P., Garber, J., Miller, K.B., Abad, M., Bruno, E.F., Carter, J.S., DavidFerdon, C., Jandasek ,B., Mennuti-Washburn ,J.E., O'Mahar, K., \& Zukerman, J. (2008). Evidence-based Assessment in Pediatric Psychology: Measures of Psychosocial Adjustment and Psychopathology. Journal of Pediatric Psychology, 33 (9) pp. 958-980.

Horn, J. L. (1965). A rationale and test for the number of factors in factor-analysis. Psychometrika, 30(2), 179-185.

Hunsley, J. y Mash, E.J. (2005). Introduction to the special section on developing guidelines for theevidence based assessment (EBA) of adult disorders. Psychological Assessment, 17, 251-255.

IBM Corporation (2012). IBM SPSS Statistics (21). Recuperado de http://www-01.ibm.com/software/es/ analytics/spss/

Janssens, A.. \& Deboutte, D. (2009) Screening for psychopathology in child welfare: the Strengths and Difficulties Questionnaire (SDQ) compared with the Achenbach System of Empirically Based Assessment (ASEBA). European Child and Adolescent Psychiatry, 18, 691-700.

Jhonston, C. \& Murray, C. (2003). Incremental validity in the psychological assessment of children and adolescents.Psychological Assessment, 15, 446-455.

Junta Nacional de Drogas (JND), Observatorio Uruguayo de Drogas (OUD). (2011). Sobre ruidos y nueces. Consumo de drogas legales e ilegales en la adolescencia.

Junta Nacional de Drogas (JND). (2012). Quinta Encuesta Nacional en Hogares sobre Consumo de Drogas. Informe de Investigación.

Kline, R.B. (2005), Principles and Practice of Structural Equation Modeling ( $2^{\text {nd }}$ Edition). New York: The Guilford Press.

Lacalle Sisteré, M. (2009). Escalas DSM en YSR y CBCL en niños y adolescentes que acuden a consultas en servicios de salud mental. Tesis Doctoral. Universidad Autónoma de Barcelona.

Laceulle, O M, Vollebergh, W. A. M. \& Ormel, J. (2015). The Structure of Psychopathology in Adolescence: Replication of a General Psychopathology Factor in the Trails Study. Clinical Psychological Science, 1-11.

Lahey, B. B., Applegate, B., Waldman, I. D., Loft, J. D., Hankin, B. L. \& Rick, J.(2004). The Structure of Child and Adolescent Psychopathology: Generating New Hypotheses. J. of Abnormal Psychology, 113(3), 358-385.

Lemos Giráldez, S, Vallejo Seco, G.\& Sandoval Mena ,M. (2002). Estructura factorial del Youth Self-Report (YSR). Psicothema, 14(4), 816-822.

Lenzenweger, M. F. \& Clarkin J. F.(2004). Major theories of personality disorder. NY: Guilford Press.

León, O.\& Montero, I. (2002). Métodos de Investigación en Psicología y Educación. 3ra Ed. Mc Graw Hill. Madrid.

Livacic-Rojas, P., Vallejo, G., \& Fernández, P. (2006). Procedimientos estadísticos alternativos para evaluar la robustez mediante diseños de medidas repetidas. $R e$ vista Latinoamericana de Psicología, 38(3), 579-598.

Llambí, C. \& Piñeiro, L. (2012). Índice de Nivel Socioeconómico INSE, Revisión anual 2012. Centro de Investigaciones Económicas (CINVE), Uruguay.

Lloret-Segura, S., Ferreres-Traver, A., \& Tomás-Marco, A. H. I. (2014). El análisis factorial exploratorio de los ítems : una guía práctica, revisada y actualizada Introducción Determinación de la adecuación del Análisis. Anales de Psicología, 30(3), 1151-1169. doi:10.6018/analesps.30.3.199361 
López Soler, C., Fernández Fernández, M., Prieto M., Alcántara, M., Castro Sáez, M. \& López Pina, J. (2012). Prevalencia de las alteraciones emocionales en una muestra de menores maltratados. Anales de psicología, 28(3), 780-788..

López Soler, C., García Montalvo, C., Pérez López, J., Brito, A., Tejerína, María \& Fernández Ros, E. (1998). Psicopatología en la adolescencia: Taxonomías empíricas, rasgos de personalidad y estrés. (Proyecto HUM96/46). Murcia, Programa Séneca del Plan Regional de Investigación, Desarrollo Tecnológico y del Conocimiento.

Lorenzo-Seva, U. (1999a). Promin: A Method for Oblique Factor Rotation. Multivariate Behavioral Research, 34(3), 347-365. doi:10.1207/S15327906MBR3403_3

Lorenzo-Seva, U. (1999b). Using Graphical Methods in Assessing Measurement Invariance in Inventory Data. Multivariate Behavioral Research, 34(908199161), 347-365. doi:10.1207/S15327906MBR3403

Lorenzo-Seva, U., \& Ferrando, P. J. (2006). FACTOR: a computer program to fit the exploratory factor analysis model. Behavior Research Methods, 38(1), 88-91. doi:10.3758/BF03192753

Lozano, L. M., García-Cueto, E. y Muñiz, J. (2008). Effect of the number of response categories on the reliability and validity of rating scales. Methodology, 4, 73-79.

Lyubomirsky, S, King, L \& Dienner E. (2005). The Benefits of Frequent Positive Affect: Does Happiness Lead to Success? Psychological Bulletin, 131(6). 803-855.

Mardia, k. v. (1970). Measures of Multivariate Skewness and Kurtosis with Principal Components. Biometrika, 57(3), 519-530.

Martínez de Salazar, A. (2001). Síndromes Empíricos en Psicopatología de la Adolescencia: Factorización de la Escala Youth Self Report de Achenbach. Tesis Doctoral: Universidad de Murcia.

Matsunaga, M. (2010). How to Factor-Analyze Your Data Right: Do's, Don'ts, and How-To's. International Journal of Psychological Research, 3(1), 97-110.

Meehl P. E (2004). What's in a Taxon? Journal of Abnormal Psychology 113, 39-43.

Meehl P. E. (2001). Comorbidity and taxometrics. Clinical Psychology. Science and Practice, 8, 507-519.

Meehl, P. E. (1999). Clarifications about taxometric method. Journal of Applied and Preventive Psychology, 8, 165-174.

Mels, C. \& Trias, D. (2014). Características preliminares del HSCL-A adaptado para adolescentes uruguayos en contexto de violencia. Ciencias Psicológicas VIII (2) 139-149.

Muñiz, J., Elosua, P. \& Hambleton, R. (2013) Directrices para la traducción y adaptación de los tests: segunda edición. Psicothema, 25(2), 151-157.

Muthén, B., du Toit, S. H. \& Spisic, D. (1997). Robust inference using weighted least squares and quadratic estimating equations in latent variable modeling with categorical and continuous outcomes. Psychometrika, $75,1-45$.

Muthén, B., \& Kaplan D. (1985). A comparison of some methodologies for the factor analysis of non-normal Likert variables. British Journal of Mathematical and Statistical Psychology, 38, 171-189.

Muthén, B., \& Kaplan, D. (1992). A comparison of some methodologies for the factor analysis of non-normal Likert variables: A note on the size of the model. British Journal of Mathematical and Statistical Psychology, 45, 19-30.
Muthén, L. K., \& Muthén, B. O. (1997). Mplus User's Guide. Psychometrika, 75, 1-45.

Muthén, L.K. and Muthén, B.O. (1998-2007). Mplus User's Guide. Fifth Edition. Los Angeles, CA: Muthén \& Muthén

Navarro-Pardo, Moral, Galan, \& Beitia (2012) Desarrollo infantil y adolescente, trastonros mentales mas frecuentes en funcion de la edad y genero. Psicothema 24 (3).

Nelson ,E., Hanna, G., Hudziak, J., Botteron, K., Heath, A. \& Todd, R. (2015) Obsessive-Compulsive Scale of the Child Behavior Checklist: Specificity, Sensitivity and Predictive Power. Recuperado http://pediatrics. aappublications.org/content/108/1/e14.full

Nunnally, J.C. y Bernstein, I.H. (1994). Psychometric theory (3rd Ed.). New Cork: Wiley.

Organización Mundial de la Salud (OMS) (2013) http:// www.who.int/features/factfiles/mental_health/mental_health_facts/es/index.html

Organización Mundial de la Salud (OMS). (1992). CIE10: Trastornos Mentales y del Comportamiento: Descripciones clínicas y pautas para el diagnóstico. Madrid: Meditor.

Organización Mundial de la Salud (OMS). (2009). Epidemiología de los Trastornos Mentales en América Latina y el Caribe. Organización Panamericana de salud. Editores: Rodríguez, J, Khon, R, Aguilar, S.

Organización Mundial de la Salud (OMS).(2013) http:// www.who.int/features/factfiles/mental_health/mental_health_facts/es/index.html

Organización Panamericana de la Salud. (1998). La Salud en las Américas, Publicación Científica, 569 (1), 193-194.

Peirano, N. (2013). Evaluación de la Resiliencia: una revisión sistemática sobre las técnicas de mayor uso. Tesis de grado en Psicología. Montevideo: Universidad Católica del Uruguay.

Pérez Algorta G. (2001). Taxonomías derivadas empíricamente de adolescentes de un instituto educativo privado. Tesis de grado en Psicología. Montevideo: Universidad Católica del Uruguay.

Pérez, E., \& Medrano, L. (2010). Análisis Factorial Exploratorio : Bases Conceptuales y Metodológicas. Revista Argentina de Ciencias del Comportamiento, 2(1), 58-66.

Rescorla L. A, Achenbach TM, Ivanova MY, Harder VS, Otten L, Bilenberg N, ... Verhulst FC. (2011). International comparisons of behavioral and emotional problems in preschool children: parents' reports from 24 societies. Journal of Clinical Child and Adolescent Psychology, 40 (3), 456 - 467.

Rescorla L.A., Ginzburg S, Achenbach TM, Ivanova MY, Almqvist F, Begovac I, ... Verhulst FC. (2013). Crossinformant agreement between parent-reported and adolescent self-reported problems in 25 societies. Journal of Clinical Child and Adolescent Psychology, 42(2), 262-73

Rescorla, L. A; Bochicchio, L.; Achenbach, T. M; Ivanova, M. Y; Almqvist, F.; Begovac, ... Verhulst, F. C. (2014). Parent-teacher agreement on children's problems in 21 societies. Journal of Clinical Child and Adolescent Psychology, 43 (4), 627-642.

Richter J, Sagatun A, Heyerdahl S, Oppedal B, Roysamb E (2011) The Strengths and Difficulties Questionnaire (SDQ) - self-report. An analysis of its structure in a multiethnic urban adolescent sample. Journal of Child Psychology and Psychiatry, 52, 1002-11

Rodríguez, M. N., \& Ruíz-Díaz, M. A. (2008). Atenuación de la asimetría y de la curtosis de las puntuaciones observadas mediante transformaciones de variables: Incidencia sobre la estructura factorial. Psicologica, 29(2), 205-227. 
Roy A k, Lopes V \& Klein R G. (2014). Disruptive Mood Dysregulation Disorder: A New Diagnostic Approach to Chronic Irritability in Youth. American Journal of Psychiatry, 171(9): 918-924.

Rutter, M. (1997) .Comorbidity: concepts, claims and choices. Criminal Behaviour and Mental Health, 7, 265-285.

Rutter, M.; Giller, H. \& Hagell, A.(2000). La conducta antisocial de los jóvenes. Madrid: Cambridge University Press.

Schmidt, N. B.; Kotov, R. \& Joiner, T. E. Jr. (2004). Taxometrics. Toward a new Diagnostic Scheme for Psychopathology. Washington, DC: American Psychological Association.

Schmitt T. A. (2011). Current methodological considerations in exploratory and confirmatory factor analysis. Journal of Psychoeducational Assessment, 29, 304-321. doi: 10.1177/0734282911406653

Schreiber, J., Nora, A., Stage, F., Barlow, E., \& King, J. (2006). Reporting Structural Equation Modeling and Confirmatory Factor Analysis Results: A Review. Journal of Educational Research, 99(6), 323-337. doi:10.3200/JOER.99.6.323-338

Timmerman, M. E. \& Lorenzo-Seva, U. (2011). Dimensionality assessment of ordered polytomous items with parallel analysis. Psychological Methods, 16(2), 209-220. doi.org/10.1037/a0023353

van der Ende, J., Verhulst, F. C., \& Tiemeier, H. (2012). Agreement of informants on emotional and behavioral problems from childhood to adulthood. Psychological Assessment, 24, 293-300

Vanderplasschen, W, Bloor, M, McKeganey, N ( 2010). LongTerm Outcomes of Aftercare Participation following Various Forms of Drug Abuse Treatment in Scotland. Journal of Drug issues. 40(3):703-728.

Veerman J. W.\& De Meyer R.(2012). Ten-Year Time Trends in Emotional and Behavioral Problems of Dutch Children Referred for Youth Care. Journal of Emotional and Behavioral Disorders, 20 (3), 184-192.
Verhulst. F.C., van der Ende, J. y Rietbergen, A. (1997) Ten years time trends of psychopathology in Dutch children and adolescents: no evidence for strong trends. Acta Psychiatry Scandinavians, 96(1),7-13.

Viola, L, Garrido, G. \& Varela, A (2007). Estudio Epidemiológico de la Salud Mental de los Niños Uruguayos entre 6 y 11 años. Montevideo: GEGA.

Wagnild, G.M. \& Young, H.M. (1993). Development and psychometric evaluation of the Resilience Scale. Journal of Nursing Measurement. Vol 1, pp165-178

Williams, B., Brown, T., \& Onsman, A. (2010). Exploratory factor analysis: A five-step guide for novices. Australasian Journal of Paramedicine, 8 (3).

Wongtongkam, N, Ward, P, Day, A, Winefield, A (2014) The influence of protective and risk factors in individual, peer and school domains on Tai adolescents alcohol and illicit drug use: A survey. Addictive Behaviours 39 1447-1451.

Wright, J. C; Zakriski, A. L. y Drinkwater, M.. (1999). Developmental Psychopathology and the Reciprocal Patterning of Behavior and Environment Distinctive Situational and Behavioral Signatures of Internalizing, Externalizing, and Mixed- Syndrome Children. Journal of Consulting and Clinical Psychology, 67(1), 95-107.

Youngstrom, E. A. (2013). Future directions in psychological assessment: combining evidence-based medicine innovations with psychology's historical strengths to enhance utility. Journal of Clinical Child Adolescent Psychology, 42 (1), 139-59.

Zubeidat, I., Fernández-Parra, Ortega J., Vallejo Miguel Á. \& Sierra J. C.(2009). Características psicosociales y psicopatológicas en una muestra de adolescentes españoles a partir del Youth Self-Report/11-18. Anales de Psicología, 25(1), 60-69.

Para citar este artículo:

Daset, L. R., Fernández-Pintos, M. E., Costa-Ball, D., López-Soler, C., \& Vanderplasschen, W. (2015). Desarrollo y validación del autoinforme de adolescentes: ADA. Ciencias Psicológicas 9(1): 85 - 104 